O. Loos

Nagoya Math. J.

Vol. 74 (1979), 23-66

\title{
ON ALGEBRAIC GROUPS DEFINED BY JORDAN PAIRS
}

\author{
OTTMAR LOOS
}

\section{Introduction}

Let $G$ be an algebraic group over a field $k$, and let $\psi$ be an action of the multiplicative group $k_{m}$ of $k$ on $G$ by automorphisms. We say $\psi$ is an elementary action if it has only the weights $0, \pm 1$; more precisely, if there exist subgroups $H, U^{+}, U^{-}$of $G$ such that (i) $H$ is fixed under $\psi$, (ii) $U^{+}$and $U^{-}$are vector groups and $\psi_{t}(x)=t^{ \pm 1} x$ for $t \in k_{m}, x \in U^{ \pm}$, (iii) $\Omega=U^{-} \cdot H \cdot U^{+}$is open in $G$, and (iv) $G$ is generated by $H, U^{+}, U^{-}$. This situation is characteristic for the complexifications of the automorphism groups of bounded symmetric domains (see, e.g., [9, 16]). A typical example is $G=\mathbf{G L}_{n}$ with (matrices being decomposed into 4 blocks) $\psi$ given by

$$
\psi_{t}\left(\begin{array}{ll}
a & b \\
c & d
\end{array}\right)=\left(\begin{array}{ll}
a & t b \\
t^{-1} c & d
\end{array}\right)
$$

If $G$ is reductive and $U^{+}$and $U^{-}$are one-dimensional, then an elementary action is essentially equivalent to an elementary system in the sense of Demazure [7, Exp. XX], with the technical difference that we consider an external torus action instead of a sub-torus of $G$ acting by conjugation.

After some preliminaries, our first goal $(\S 4)$ is to find relations describing the structure of $G$ in terms of the generators $H, U^{+}, U^{-}$. Since $H$ normalizes $U^{+}$and $U^{-}$this essentially amounts to a formula expressing products in $U^{+} \cdot U^{-}$in terms of their components in $\Omega=U^{-} \cdot H \cdot U^{+}$. In more detail, let $\mathfrak{B}^{ \pm}$be the Lie algebra of $U^{ \pm}$. Then there are $\psi$-equivariant isomorphism exp: $\mathfrak{B}^{ \pm} \rightarrow U^{ \pm}$, and there is a unique Jordan pair structure on $\mathfrak{B}=\left(\mathfrak{B}^{+}, \mathfrak{B}^{-}\right)$such that, for $x \in \mathfrak{B}^{+}, y \in \mathfrak{B}^{-}$, the product $\exp (x) \cdot \exp (y)$ belongs to $\Omega$ if and only if $(x, y)$ is quasi-invertible, and in this case

$$
\exp (x) \cdot \exp (y)=\exp \left(y^{x}\right) \cdot b(x, y) \cdot \exp \left(x^{y}\right) \text {. }
$$

Here $x^{y}, y^{x}$ denotes the quasi-inverse in $\mathfrak{B}$ and $b$ is a morphism from the set of quasi-invertible pairs of $\mathfrak{B}$ into $H$ which has properties analogous to the "Bergmann transformations" $B(x, y)$ of a Jordan pair. The formula 
$(*)$ is the higher-dimensional analogue of Demazure's formula $(F)$ ([7, Exp. XX, Th. 2.1]). In case $U^{+}$and $U^{-}$are conjugate by an element of $G^{0}$, one can show that the Jordan pair $\mathfrak{B}$ contains invertible elements and hence is the Jordan pair defined by a (not canonically determined) unital quadratic Jordan algebra; see Borel-Tits [4, § 5] and Springer [20, 2.21-2.26].

Next we show how to reconstruct $G$, given $\mathfrak{B}$ and $H$. The necessary ingredients for this are an action $\rho$ of $H$ on $\mathfrak{B}$ by automorphisms and a morphism $b$ from the quasi-invertible pairs of $\mathfrak{B}$ into $H$, satisfying suitable conditions (5.1). Then for every "Jordan system" ( $\mathfrak{B}, H, \rho, b)$ there exists an "elementary system" $(G, \psi)$, and this establishes an equivalence of categories. In case $H$ is the automorphism group of $\mathfrak{B}$ and $\rho=I d$, and under restrictions on $k$ and $\mathfrak{B}$, Koecher proved the existence of $G$ by realizing it as a group of birational transformations; see $[11,12,13]$. The proof given here (5.2-5.9) is more direct but also more computational. The Lie algebra of $G$ was studied extensively by Tits [21], Koecher [10], and Meyberg [17].

In $\S 6$, we prove that the unipotent radical of $G$ and the Jacobson radical of $\mathfrak{B}$ are related by the formula

$$
R_{u}(G)=N^{-} \cdot R_{u}(H) \cdot N^{+}
$$

where $N^{ \pm}=\exp \left(\operatorname{Rad} \mathfrak{B}^{ \pm}\right)$. We also show that the automorphism group of a separable Jordan pair is reductive and its identity component consists of inner automorphisms, a result due to Springer [20] in the Jordan algebra case. Finally, we give a description of the group $G(k)$ of $k$-rational points by generators and relations.

The desire to replace the base field $k$ by an arbitrary base ring leads naturally to considering group schemes and Jordan pairs which are finitely generated and projective $k$-modules. In view of recent work of H. P. Petersson [18] on orders in Jordan pairs over quotient fields of Dedekind rings, this degree of generality seems not without interest. It turns out that the formal theory of $\S 3-\S 5$ is easier to handle in the framework of group sheaves (in the flat topology). Representability questions are then treated separately; if the base ring is Dedekind, they have satisfactory answers (5.13). We have therefore adopted this point of view, in particular, since it involves very little extra effort as compared to a more classical approach.

The author wishes to express his thanks to P. Gabriel for a stimulating conversation. Also, the hospitality of the University of California at 
Irvine during the final preparation of the manuscript is gratefully acknowledged.

\section{$\S 1 . \quad$ Notations and Preliminaries}

1.1. Let $k$ denote a commutative base ring and $k$-alg the category of commutative $k$-algebras. We follow the notations and conventions of [5]. In particular, schemes are considered as special $k$-functors (covariant functors from $k$-alg into sets). If $X$ is a $k$-functor we write $x \in X$ for $x \in X(R), R \in k$-alg. The base extension of $X$ from $k$ to $K$ is denoted by $X_{K}$ or $X \otimes_{k} K$. A sheaf is a $k$-functor which is a sheaf in the flat (= fppf) topology. We refer to [5, p. 10, p. 50] for the notion of open (closed) subfunctor. It is easily seen that an open (closed) subfunctor of a sheaf is itself a sheaf. A $k$-functor $X$ is called separated if the diagonal in $X \times X$ is closed. The following result can be proved along the lines of $[5$, p. 296].

1.2. Lemma. Let

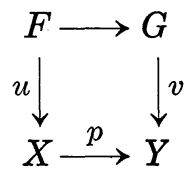

be a Cartesian square of sheaves where $p$ is an epimorphism of sheaves. Then $u$ is an open (closed) imbedding if and only if $v$ is.

1.3. Corollary. Let $p: X \rightarrow Y$ be an epimorphism of sheaves and $E$ $=X \times{ }_{Y} X \subset X \times X$ the equivalence relation defined by $p$. Then $Y$ is separated if and only if $E$ is closed in $X \times X$.

This follows by considering the Cartesian square

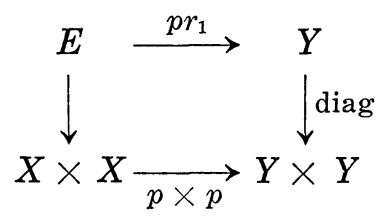

1.4. A subfunctor $U$ of a $k$-functor $X$ is called dense if, for all open $V \subset X$, and all closed $Z \subset V$ such that $Z \supset U \cap V$ we have $Z=V$, and this property remains valid in all scalar extensions. If $X$ is a scheme then this notion of dense is the same as "universally schematically dense" 
(cf. EGA IV, 11.10). The following lemma will be used often (cf. SGA3, Exp. XVIII, 1.7, and EGA IV, 11.10.10).

1.5. Lemma. Let $X$ be a smooth separated finitely presented $k$-scheme with connected non-empty fibres, and let $U$ be an open subscheme of $X$. Then the following conditions are equivalent.

(i) $U$ is dense in $X$.

(ii) There exists a fppf extension $R$ of $k$ such that $U(R) \neq \varnothing$.

(iii) $U(K) \neq \varnothing$ for all algebraically closed fields $K \in k$-alg.

1.6. Lemma (Cf. SGA3, Exp. XVIII, Prop. 1.1). Let $U$ be a dense subfunctor of $X$.

(a) $U_{R}$ is dense in $X_{R}$, for all $R \in k$-alg.

(b) If $U \subset U^{\prime} \subset X$ then $U^{\prime}$ is dense in $X$.

(c) If $V$ is open in $X$ then $U \cap V$ is dense in $V$.

(d) If $V \subset U$ is dense in $U$ then $V$ is dense in $X$.

(e) If $U^{\prime} \subset X$ is open and dense then $U \cap U^{\prime}$ is dense in $X$.

(f) $U \times Y$ is dense in $X \times Y$, for any $k$-functor $Y$.

(g) If $f, g: X \rightarrow Y$ are morphisms into a separated $k$-functor $Y$ which agree on $U$ then $f=g$.

The proofs are mostly straightforward and are omitted.

1.7. Lemma. Let $p: X \rightarrow Y$ be an epimorphism of sheaves, and let $U$ be a subfunctor of $Y$ such that $p^{-1}(U)$ is dense in $X$. Then $U$ is dense.

Proof. Let $V \subset Y$ be open, $Z \subset V$ closed, and assume $U \cap V \subset Z$. Then $p^{-1}(V)$ is open in $X, p^{-1}(Z) \subset p^{-1}(V)$ is closed, and $p^{-1}(U) \cap p^{-1}(V)$ $\subset p^{-1}(Z)$. Hence $p^{-1}(Z)=p^{-1}(V)$. Now $p: p^{-1}(V) \rightarrow V$ is an epimorphism of sheaves, and therefore $Z$ contains the image sheaf of $p^{-1}(V)$ under $p$ which is $V$.

1.8. A $k$-group functor is a covariant functor from $k$-alg into the category of groups. If $G$ is a $k$-group sheaf and $A$ and $B$ are subsheaves of $G$ then $A \cdot B$ denotes the image sheaf of $A \times B$ under multiplication. The multiplicative group of $k$ is denoted by $k_{m}$, the additive group of a finitely generated projective $k$-module $\mathfrak{M}$ by $\mathfrak{M}_{a}$. Thus $\mathfrak{M}_{a}(R)=\mathfrak{M}_{R}=$ $\mathfrak{M} \otimes_{k} R$, for all $R \in k$-alg. The Lie algebra of a $k$-group functor $G$ is denoted by Lie $(G)$. We follow [7, p. 209] and write $e^{\varepsilon x} \in G(k(\varepsilon))$ for $x \in \operatorname{Lie}(G)$ (where $k(\varepsilon)$ is the ring of dual numbers). If $G=\mathfrak{M}_{a}$ is a 
vector group we identify $\operatorname{Lie}\left(\mathfrak{M}_{a}\right)$ with $\mathfrak{M}$ and set $e^{\varepsilon x}=\varepsilon x$. If $G \subset \mathbf{G L}(\mathfrak{M})$ is linear then we identify Lie $(G)$ with the set of $x \in$ End (M) such that $e^{\varepsilon x}$ $=\mathrm{Id}+\varepsilon x \in G(k(\varepsilon))$. (Here $\mathbf{G L}(\mathfrak{M})$ is the $k$-group functor $R \mapsto \mathrm{GL}\left(\mathfrak{M}_{R}\right)$ ). The adjoint representation of $G(k)$ on Lie $(G)$ is defined by $\operatorname{Int}(g) e^{\varepsilon x}=e^{\varepsilon \text { Adg.x }}$, where $\operatorname{Int}(g) h=g h g^{-1}$.

\section{§2. Jordan Pairs}

2.1. Let $\mathfrak{B}^{+}$and $\mathfrak{B}^{-}$be finitely generated and projective $k$-modules, and let $Q_{+}: \mathfrak{B}^{+} \rightarrow \operatorname{Hom}\left(\mathfrak{B}^{-}, \mathfrak{B}^{+}\right)$and $Q_{-}: \mathfrak{B}^{-} \rightarrow \operatorname{Hom}\left(\mathfrak{B}^{+}, \mathfrak{B}^{-}\right)$be quadratic maps. For $\sigma= \pm$ define trilinear compositions $\mathfrak{B}^{\sigma} \times \mathfrak{B}^{-\sigma} \times \mathfrak{B}^{\sigma} \rightarrow \mathfrak{B}^{\sigma}$, $(x, y, z) \mapsto\{x y z\}$, and bilinear maps $D_{o}: \mathfrak{B}^{\sigma} \times \mathfrak{B}^{-\sigma} \rightarrow$ End $\left(\mathfrak{B}^{\sigma}\right)$ by

$$
\{x y z\}=D_{\sigma}(x, y) z=Q_{\sigma}(x, z) y=Q_{\sigma}(x+z) y-Q_{\sigma}(x) y-Q_{\sigma}(z) y .
$$

The pair $\mathfrak{B}=\left(\mathfrak{B}^{+}, \mathfrak{B}^{-}\right)$together with the quadratic maps $\left(Q_{+}, Q_{-}\right)$is called a Jordan pair if the following identities hold in all base ring extensions.

$$
\begin{aligned}
& D_{\sigma}(x, y) Q_{\sigma}(x)=Q_{\sigma}(x) D_{-\sigma}(y, x), \\
& D_{\sigma}\left(Q_{\sigma}(x) y, y\right)=D_{\sigma}\left(x, Q_{-\sigma}(y) x\right), \\
& Q_{\sigma}\left(Q_{\sigma}(x) y\right)=Q_{\sigma}(x) Q_{-\sigma}(y) Q_{\sigma}(x) .
\end{aligned}
$$

A homomorphism $h: \mathfrak{B} \rightarrow \mathfrak{W}$ of Jordan pairs is a pair $h=\left(h_{+}, h_{-}\right)$of $k$-linear maps, $h_{\sigma}: \mathfrak{B}^{\sigma} \rightarrow \mathfrak{W}^{\sigma}$, such that $h_{\sigma} Q_{\sigma}(x)=Q_{\sigma}\left(h_{\sigma}(x)\right) h_{-\sigma}$. The automorphism group of $\mathfrak{B}$ is denoted by Aut $(\mathfrak{B})$. The opposite of $\mathfrak{B}$ is $\mathfrak{B}^{\mathrm{op}}=\left(\mathfrak{B}^{-}, \mathfrak{B}^{+}\right)$ with quadratic maps $\left(Q_{-}, Q_{+}\right)$. A standard example of a Jordan pair is $\mathfrak{B}^{+}=\mathfrak{B}^{-}=M_{p, q}(k), p \times q$-matrices, with $Q_{ \pm}(x) y=x \cdot{ }^{t} y \cdot x$. For details see [15].

2.2. The quasi-inverse $([15, \S 3])$. Let $\mathfrak{B}$ be a Jordan pair. Following the convention of $[15,2.0]$, we omit the index $\sigma$ in $D_{\sigma}$ and $Q_{\sigma}$ and write $Q_{x} y$ for $Q(x) y$. For $x \in \mathfrak{B}^{+}, y \in \mathfrak{B}^{-}$define $B(x, y) \in \operatorname{End}\left(\mathfrak{B}^{+}\right)$and $B(y, x) \in$ End $\left(\mathfrak{B}^{-}\right)$by

$$
B(x, y)=\operatorname{Id}-D(x, y)+Q_{x} Q_{y}, \quad B(y, x)=\operatorname{Id}-D(y, x)+Q_{y} Q_{x} .
$$

The pair $(x, y) \in \mathfrak{B}^{+} \times \mathfrak{B}^{-}$is called quasi-invertible if there exists $z \in \mathfrak{B}^{+}$ such that

$$
B(x, y) z=x-Q_{x} y \quad \text { and } \quad B(x, y) Q_{z} y=Q_{x} y .
$$

This is the case if and only if $B(x, y)$ is invertible, and thus 


$$
z=x^{y}=B(x, y)^{-1}\left(x-Q_{x} y\right),
$$

called the quasi-inverse of $(x, y)$. We denote by $W \subset \mathfrak{B}_{a}^{+} \times \mathfrak{B}_{a}^{-}$the open dense subscheme of quasi-invertible pairs. In the example above, $(x, y)$ is quasi-invertible if and only if $1-x \cdot{ }^{t} y$ is invertible, and $x^{y}=$ $\left(1-x \cdot{ }^{t} y\right)^{-1} \cdot x$. If $(x, y)$ is quasi-invertible then

$$
\beta(x, y)=\left(B(x, y), B(y, x)^{-1}\right)
$$

is an automorphism of $\mathfrak{B}$, called the inner automorphism defined by $(x, y)$.

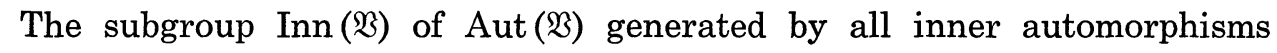
is normal and called the inner automorphism group.

2.3. The automorphism group functor of $V$ is defined by

$$
\operatorname{Aut}(\mathfrak{V})(R)=\operatorname{Aut}\left(\mathfrak{V}_{R}\right) \text {, }
$$

for all $R \in k$-alg. We show that Aut(B) is an affine finitely presented group scheme. Let $x_{1}^{\sigma}, \cdots, x_{n}^{\sigma}$ be a set of generators for the $k$-module $\mathfrak{B}^{\sigma}(\sigma= \pm)$. Then $h=\left(h_{+}, h_{-}\right) \in \mathrm{GL}\left(\mathfrak{B}^{+}\right) \times \mathrm{GL}\left(\mathfrak{B}^{-}\right)$belongs to Aut () if and only if

$$
\begin{aligned}
& h_{\sigma} Q\left(x_{i}^{\sigma}\right)=Q\left(h_{\sigma}\left(x_{i}^{\sigma}\right)\right) h_{-\sigma}, \\
& h_{\sigma} D\left(x_{i}^{\sigma}, x_{j}^{-\sigma}\right)=D\left(h_{\sigma}\left(x_{i}^{\sigma}\right), h_{-\sigma}\left(x_{j}^{-\sigma}\right)\right) h_{\sigma},
\end{aligned}
$$

for $i, j=1, \cdots, n, \sigma= \pm$. Since this remains true in any base ring extension, Aut $(\mathfrak{B})$ is the closed subscheme of the affine finitely presented $k$-scheme $\mathbf{G L}\left(\mathfrak{B}^{+}\right) \times \mathbf{G L}\left(\mathfrak{B}^{-}\right)$defined by finitely many equations, and is therefore itself an affine finitely presented $k$-scheme $([5, \mathrm{I}, \S 3])$.

The derivation algebra of $\mathfrak{B}$ is $\operatorname{Der}(\mathfrak{B})=\operatorname{Lie}($ Aut $(\mathfrak{B})$ ). From 1.8 it follows easily that $\Delta=\left(\Delta_{+}, \Delta_{-}\right) \in \operatorname{End}\left(\mathfrak{B}^{+}\right) \times \operatorname{End}\left(\mathfrak{B}^{-}\right)$is a derivation of $\mathfrak{B}$ if and only if

$$
\Delta_{\sigma} Q_{x} y=\left\{\Delta_{\sigma} x, y, x\right\}+Q_{x} \cdot \Delta_{-o} y,
$$

for all $x \in \mathfrak{B}^{\sigma}, y \in \mathfrak{B}^{-\sigma}, \sigma= \pm$. Finally we note that there is a central homomorphism $\gamma: k_{m} \rightarrow$ Aut () given by

$$
\gamma(t)=\left(t \cdot \operatorname{Id}, t^{-1} \cdot \mathrm{Id}\right) .
$$

2.4. Let Inn (B) be the $k$-group sheaf associated with the $k$-group

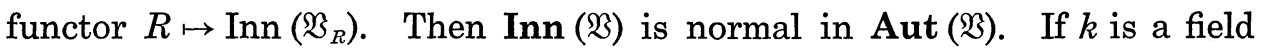
then Inn $(\mathfrak{B})$ is a smooth connected algebraic $k$-group since it is generated by $\beta(W)$ ([SGA3, Exp. $\mathrm{VI}_{B}$, No. 7], see also [2, p. 106]). For $(x, y) \in \mathfrak{B}^{+} \times \mathfrak{B}^{-}$, 


$$
\delta(x, y)=(D(x, y),-D(y, x))
$$

is a derivation of $\mathfrak{B}$, called the inner derivation defined by $(x, y)$. The

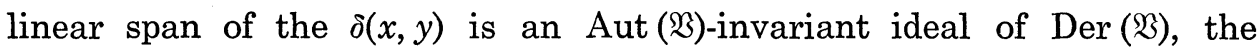

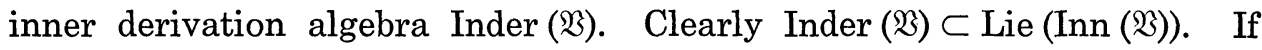
$k$ is a field of characteristic zero then it can be shown that equality holds.

2.5. Let $\mathfrak{A}$ be a unital quadratic Jordan algebra over $k$ which is finitely generated and projective as a $k$-module. Then $\mathfrak{U}$ defines a Jordan pair $\mathfrak{B}=(\mathfrak{A}, \mathfrak{U})$ by setting $\mathfrak{B}^{+}=\mathfrak{B}^{-}=\mathfrak{A}$ and $Q(x) \cdot y=P(x) \cdot y$ where $P$ denotes the quadratic representation of $A$. This establishes a one-to-one correspondence between Jordan algebras "up to isotopy" and Jordan pairs containing invertible elements $([15,1.11])$. Let $\operatorname{Str}(\mathfrak{U})$ be the structure group of $\mathfrak{A}$; i.e., the group of all $g \in \mathrm{GL}$ (I) such that $P(g x)=g P(x) g^{\sharp}$ for some $g^{\sharp} \in \mathrm{GL}(\mathfrak{U})$ and all $x \in A$. Then $g^{\sharp} \in \operatorname{Str}(\mathfrak{U})$ and the map $g \mapsto g^{\sharp-1}$ is an automorphism of period 2 of $\operatorname{Str}(\mathfrak{U})$. The inner structure group is the subgroup Instr (I) of $\operatorname{Str}(\mathfrak{U})$ generated by all $P(x), x$ invertible. The structure group functor $\operatorname{Str}(\mathfrak{U})$ is defined by $\operatorname{Str}(\mathfrak{U})(R)=\operatorname{Str}\left(\mathfrak{U}_{R}\right)$ and we denote by Instr ( $\mathfrak{l})$ the $k$-group sheaf generated by the $k$-group functor $R \mapsto \operatorname{Instr}\left(\mathfrak{U}_{R}\right)$.

2.6. Proposition. The map $f: g \mapsto\left(g, g^{\#-1}\right)$ is an isomorphism of

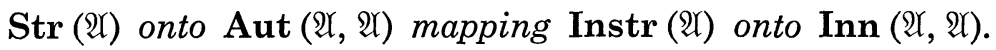

Proof. The first statement follows easily from the definitions (cf.

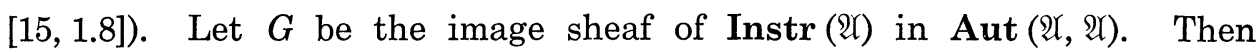
$G \subset \operatorname{Inn}(\mathfrak{U}, \mathfrak{X})$ since $P(x)=B\left(x, x^{-1}+e\right)$ and thus $f(P(x))=\beta\left(x, x^{-1}+e\right)$ (here $e$ is the unit element of $\mathfrak{2}$ ). Since everything is compatible with base extension, the converse inclusion will follow if we show: for every quasi-invertible $(x, y) \in \mathfrak{U} \times \mathfrak{U}$ there exists a fppf extension $R$ of $k$ such that $B(x, y) \in \operatorname{Instr}\left(\mathfrak{U}_{R}\right)$. If $x$ is invertible then $B(x, y)=P(x) P\left(x^{-1}-y\right) \in$ Instr (II) (cf. [15, 1.12]). If not, let $U \subset \mathfrak{U}_{a}$ be the open subscheme defined by

$$
U(R)=\left\{z \in \mathfrak{U}_{R} \mid(x+z, y) \text { quasi-invertible }\right\},
$$

and let $U^{\prime}=U \cap I \cap(-x+I)$ where $I$ is the open dense subscheme of invertible elements of $\mathfrak{A}$. Then $U$ is dense since $0 \in U(k)$, and so are $I$ and the translate $-x+I$. By 1.5 and 1.6, $U^{\prime}$ is dense and hence there exists a fppf extension $R$ of $k$ such that $U^{\prime}(R) \neq \varnothing$. Picking $z \in U^{\prime}(R)$ we have by $\left[15\right.$, p. 25, JP34] that $B(x, y)=B\left(z, y^{x}\right)^{-1} B(x+z, y) \in \operatorname{Instr}\left(\mathfrak{A}_{R}\right)$. 


\section{§3. Elementary torus actions}

3.1. Let $G$ be a separated $k$-group sheaf, and let $\psi$ be an action of the multiplicative group $k_{m}$ on $G$ by automorphisms. Thus for every invertible $t \in R, R \in k$-alg we have an automorphism $\psi_{t}$ of $G_{R}$ varying functorially with $R$ and satisfying $\psi_{s} \psi_{t}=\psi_{s t}$. The action $\psi$ is called elementary, and the pair $(G, \psi)$ is called an elementary system if there exist subgroup sheaves $H, U^{+}, U^{-}$of $G$ with the following properties.

(i) $H$ is fixed under $\psi$.

(ii) $U^{+}$and $U^{-}$are vector groups on which $\psi$ acts by scalar multiplication (resp. the inverse of scalar multiplication).

More precisely: there exist finitely generated and projective $k$-modules $\mathfrak{M}^{ \pm}$and isomorphisms $f_{ \pm}: \mathfrak{M}_{a}^{ \pm} \rightarrow U^{ \pm}$such that $f_{+}(t x)=\psi_{t} \cdot f_{+}(x), f_{-}\left(t^{-1} y\right)=$ $\psi_{t} \cdot f_{-}(y)$, for all $t \in k_{m}, x \in \mathfrak{M}_{a}^{+}, y \in \mathfrak{M}_{a}^{-}$.

(iii) $\Omega=U^{-} \cdot H \cdot U^{+}$is open in $G$.

(iv) $G$ is generated (as a $k$-group sheaf) by $H, U^{+}, U^{-}$.

We will show later that $H, U^{+}, U^{-}$are uniquely determined by these conditions. Clearly, if $(G, \psi)$ is an elementary system so is any base extension $\left(G_{K}, \psi_{K}\right)$. A homomorphism $f:(G, \psi) \rightarrow\left(G^{\prime}, \psi^{\prime}\right)$ is a group homomorphism $f: G \rightarrow G^{\prime}$ compatible with $\psi$ and $\psi^{\prime}$. If $\psi$ is an elementary action so is $\psi^{-1}$ defined by $\psi_{t}^{-1}=\psi_{t-1}$. This just amounts to interchanging $U^{+}$and $U^{-}$and replacing $\Omega$ by $\Omega^{-1}$.

3.2. Example. Let $G=\mathbf{G L}_{n}$ and divide a $n \times n$-matrix $g$ into 4 blocks:

$$
g=\left(\begin{array}{ll}
a & b \\
c & d
\end{array}\right)
$$

of size $p \times p, p \times q, q \times p, q \times q$, where $p+q=n$. Let

$$
\psi_{t}(g)=\left(\begin{array}{ll}
a & t b \\
t^{-1} c & d
\end{array}\right)
$$

Then $\psi_{t}$ is an action which is elementary. Indeed, let $H$ (resp. $U^{+}, U^{-}$) consist of all matrices of the form

$$
\left(\begin{array}{ll}
a & 0 \\
0 & d
\end{array}\right), \quad\left(\operatorname{resp} .\left(\begin{array}{ll}
1 & b \\
0 & 1
\end{array}\right),\left(\begin{array}{ll}
1 & 0 \\
c & 1
\end{array}\right)\right)
$$

then $\Omega$ consists of all matrices (1) where $a$ is invertible, and is therefore open. Since $G$ is a smooth separated finitely presented group scheme with 
connected fibres, $\Omega$ is dense in $G$. Let $g \in G(R)$. Then $\Omega_{R} \cap g \cdot \Omega_{R}^{-1}$ is open and dense by 1.6 , and by 1.5 there exists a fppf extension $S$ of $R$ such that $\left(\Omega_{R} \cap g \cdot \Omega_{R}^{-1}\right)(S) \neq \varnothing$; i.e., $g \in \Omega(S) \cdot \Omega(S)$. This proves (iv).

Remark. By the same argument, (iv) follows from (iii) whenever $G$ is a smooth finitely presented group scheme with connected fibres.

Along the same lines, one has elementary actions on the orthogonal and symplectic groups $\mathbf{O}_{2 n} \subset \mathbf{G L}_{2 n}$ and $\mathbf{S p}_{n} \subset \mathbf{G L}_{2 n}$. Again, $\psi$ is defined by (2), and $H$ is isomorphic with $\mathbf{G L}_{n}$ imbedded into $\mathbf{G L}_{2 n}$ via

$$
a \mapsto\left(\begin{array}{cc}
a & 0 \\
0 & t_{a}-1
\end{array}\right)
$$

The groups $U^{ \pm}$are isomorphic with the additive group of alternating (symmetric) $n \times n$-matrices.

3.3. Lemma. Let $\psi$ be an elementary action, and let $\mathfrak{B}^{ \pm}$be the Lie algebra of $U^{ \pm}$. Then there exist unique isomorphisms

$$
\exp : \mathfrak{B}_{a}^{ \pm} \rightarrow U^{ \pm}
$$

such that

$$
\begin{gathered}
\text { Lie }(\exp )=\operatorname{Id} ; \quad \text { i.e., } \quad \exp (\varepsilon x)=e^{\varepsilon x}, \\
\psi_{t}(\exp x)=\exp \left(t^{ \pm 1} x\right) \\
\operatorname{Lie}\left(\psi_{t}\right) \cdot x=t^{ \pm 1} \cdot x
\end{gathered}
$$

for all $t \in k_{m}, x \in \mathfrak{B}_{a}^{ \pm}$.

Proof. Let $f_{+}: \mathfrak{M}_{a}^{+} \rightarrow U^{+}$be as in 3.1 (ii). By the convention of 1.8, Lie $\left(f_{+}\right): \mathfrak{M}^{+} \rightarrow \mathfrak{B}^{+}$is an isomorphism of $k$-modules. Define $\exp (x)=$ $f_{+}\left(\operatorname{Lie}\left(f_{+}\right)^{-1} x\right)$. One checks easily that (1) and (2) hold, and (3) follows from (1) and (2). To prove unicity, assume that $\exp ^{\prime}$ has the same properties. Then $h=\left(\exp ^{\prime}\right)^{-1} \circ \exp : \mathfrak{B}_{a}^{+} \rightarrow \mathfrak{B}_{a}^{+}$is an isomorphism which is by (2) homogeneous of degree one, and therefore induced from a linear isomorphism of $k$-modules. By (1), this isomorphism is the identity. For exp: $\mathfrak{B}_{a}^{-} \rightarrow U^{-}$the proof is analogous.

3.4. Lemma. The map $U^{-} \times H \times U^{+} \rightarrow G$ given by multiplication is an open imbedding.

Proof. We only have to show that this map is a monomorphism. 
Using the fact that $U^{+}$and $U^{-}$are subgroups and everything is compatible with base extension, this reduces to: if

$$
\exp (y) \cdot h \cdot \exp (x)=h^{\prime}
$$

for $x \in \mathfrak{B}^{+}, y \in \mathfrak{B}^{-}, h, h^{\prime} \in H(k)$ then $h=h^{\prime}$ and $x=y=0$. Applying $\psi_{t}$ to (1) we get

$$
\exp \left(t^{-1} y\right) \cdot h \cdot \exp (t x)=h^{\prime},
$$

for all $t \in k_{m}$. In particular, for $t=1+\varepsilon \in k(\varepsilon)$ (dual numbers) (2) implies, in view of 3.3 ,

$$
\begin{aligned}
h^{\prime} & =\exp ((1-\varepsilon) y) \cdot h \cdot \exp ((1+\varepsilon) x) \\
& =\exp (-\varepsilon y) \cdot \exp (y) \cdot h \cdot \exp (x) \cdot \exp (\varepsilon x)=e^{-\varepsilon y} \cdot h^{\prime} \cdot e^{\varepsilon x},
\end{aligned}
$$

and hence $e^{\varepsilon y}=h^{\prime} e^{\varepsilon x} h^{\prime-1}=e^{\varepsilon \operatorname{Ad} h^{\prime} \cdot x}$; i.e., $y=\operatorname{Ad} h^{\prime} \cdot x$. Applying Lie $\left(\psi_{t}\right)$ to this we get, by 3.3.3, $t^{-1} y=\operatorname{Ad} h^{\prime} \cdot t x=t \cdot \operatorname{Ad} h^{\prime} \cdot x=t y$, for all $t \in k_{m}$. This implies $y=0$. Hence also $x=0$ and $h=h^{\prime}$.

3.5. Lemma. Let $f:(G, \psi) \rightarrow\left(G^{\prime}, \psi^{\prime}\right)$ be a homomorphism of elementary systems. Then $f$ maps $U^{ \pm}$into $U^{\prime \pm}$, and

$$
f(\exp (x))=\exp ^{\prime}(\operatorname{Lie}(f)(x))
$$

for all $x \in \mathfrak{B}_{ \pm}$.

Proof. Define a morphism $\varphi: \mathfrak{V}_{a}^{+} \rightarrow G^{\prime}$ by $\varphi(x)=f(\exp x)$. Then

$$
\varphi(t x)=\psi_{t}^{\prime} \cdot(\varphi(x)),
$$

for $t \in k_{m}$. Let $X=\varphi^{-1}\left(\Omega^{\prime}\right) \subset \mathfrak{B}_{a}^{+}$. Then $X$ contains the zero section, is open, and (2) implies that it is invariant under $k_{m}$ acting on $\mathfrak{B}_{a}^{+}$by scalar multiplication. It follows that $X=\mathfrak{B}_{a}^{+}$. By 3.4, we can write

$$
\varphi(x)=\exp ^{\prime}\left(g_{-}(x)\right) \cdot g_{0}(x) \cdot \exp ^{\prime}\left(g_{+}(x)\right)
$$

with unique morphisms $g_{ \pm}: \mathfrak{B}_{a}^{+} \rightarrow \mathfrak{B}_{a}^{\prime \pm}$ and $g_{0}: \mathfrak{B}_{a}^{+} \rightarrow H^{\prime}$. From (2) and (3) we get the formulas

$$
\begin{gathered}
g_{ \pm}(t x)=t^{ \pm 1} g_{ \pm}(x), \\
g_{0}(t x)=g_{0}(x),
\end{gathered}
$$

for all $t \in k_{m}$. Hence $g_{0}$ and $g_{-}$are constant equal to 1 resp. 0 , and $g_{+}$ is linear (cf. [19]). By 3.3.1, $g_{+}=\operatorname{Lie}(f) \mid \mathfrak{B}^{+}$. The argument for $\mathfrak{B}^{-}$is similar. 
3.6. Proposition. (a) The subgroups $U^{+}$and $U^{-}$are uniquely determined.

(b) $H$ normalizes $U^{+}$and $U^{-}$, and exp is $H$-equivariant:

$$
h \cdot \exp (x) h^{-1}=\exp (\operatorname{Ad} h \cdot x),
$$

for all $x \in \mathfrak{B}_{a}^{ \pm}, h \in H$.

$$
H \cap U^{+} \cdot U^{-} \cdot U^{+}=\{1\} .
$$

Proof. (a) This follows from 3.5, applied to $f=I d$. (b) Apply 3.5 to the homomorphism Int $(h):\left(G_{R}, \psi_{R}\right) \rightarrow\left(G_{R}, \psi_{R}\right)$, for any $h \in H(R), R \in k$-alg. (c) Let $h \in\left(H \cap U^{+} U^{-} U^{+}\right)(R)$. Then $h=u v w$ with $u, w \in U^{+}(S), v \in U^{-}(S)$ for some fppf extension $S$ of $R$. Hence $h^{-1} u h \in U^{+}(S)$ and $u^{-1} h=h\left(h^{-1} u^{-1} h\right)$ $=v w$. By 3.4, $h=1$.

3.7. Lemma. Let $u, w \in U^{-}(k)$ and $v \in U^{+}(k)$. Then there exists an fppf extension $R$ of $k$ and $x \in U^{+}(R)$ such that $x u^{-1}$ and $x v w$ belong to $\Omega(R)$.

Proof. Let $X=U^{+} \cap(\Omega \cdot u)$ and $Y=U^{+} \cap\left(\Omega w^{-1} v^{-1}\right)$. Then $X$ and $Y$ are open in $U^{+}$, and they are dense since $1 \in X(k)$ and $v^{-1} \in Y(k)$. Therefore $X \cap Y$ is dense, and there exists an fppf extension $R$ of $k$ such that $X(R) \cap Y(R) \neq \varnothing$ (cf. 1.5, 1.6). The lemma follows.

3.8. Proposition. $G=H \cdot U^{+} \cdot U^{-} \cdot U^{+}=U^{+} \cdot \Omega$, and $\Omega$ is dense in $G$.

Proof. Let $G^{\prime}=U^{+} \cdot \Omega=H \cdot U^{+} \cdot U^{-} \cdot U^{+}$. Then $G^{\prime}$ is a subgroup sheaf of $G$. Indeed, in view of $3.6(\mathrm{~b})$, this amounts to showing that $U^{-} \cdot U^{+} \cdot U^{-}$ $\subset G^{\prime}$. After a base extension, it suffices to show that, for all $u, w \in U^{-}(k)$, $v \in U^{+}(k)$, there exists a fppf extension $R$ of $k$ such that $u v w \in G^{\prime}(R)$. Picking $x$ as in 3.7, we have $u v w=\left(u x^{-1}\right)(x v w) \in \Omega^{-1}(R) \Omega(R)=U^{+}(R) \Omega(R)$ $\subset G^{\prime}(R)$. Since $G$ is generated by $H, U^{+}$, and $U^{-}$, we have $G=G^{\prime}$. Let $Y \subset U^{+} \times U^{-}$be the inverse image of $\Omega$ under the multiplication map $U^{+} \times U^{-} \rightarrow G$. Then $Y$ is open and dense, and the inverse image of $\Omega$ under the epimorphism of sheaves $U^{+} \times U^{-} \times H \times U^{+} \rightarrow G$ is $Y \times H \times U^{+}$. By 1.6(f) and 1.7, $\Omega$ is dense in $G$.

3.9. Let $G_{i n}$ be the subgroup sheaf of $G$ generated by $U^{+}$and $U^{-}$. Then by 3.6(b), $G_{i n}$ is normal in $G$, and clearly stable under $\psi$. Since $\Omega \cap G_{i n}=U^{-} \cdot\left(H \cap G_{i n}\right) \cdot U^{+}$is open in $G_{i n}$ we see that $\psi$ induces an elementary action on $G_{i n}$. By 3.8 we have $G=H \cdot G_{i n}$. If $k$ is a field and $G$ is an algebraic $k$-group then by $\mathrm{SGA} 3, \operatorname{Exp} \mathrm{VI}_{\mathrm{B}}$, No. $7, G_{i n}$ is a smooth 
connected algebraic $k$-group. Finally, we give the following criterion for a $k_{m}$-action to be elementary.

3.10. Proposition. Let $G$ be an affine finitely presented group scheme with connected fibres, and assume that either $G$ is reductive or $k$ is a field of characteristic 0 . Then an action $\psi$ of $k_{m}$ on $G$ by automorphisms is elementary if (and only if) $\psi$ has at most the weights $0, \pm 1$ on $g=\operatorname{Lie}(G)$.

Proof. The "only if" is immediate from 3.4. Let $\hat{G}=G \rtimes k_{\text {in }}$ be the semidirect product with $k_{m}$ acting on $G$ via $\psi$, and let $S=\{1\} \times k_{m} \subset \hat{G}$. Identify the character group of $S$ with $Z$, and let $H$ be the centralizer of $S$ in $G$. We have the decomposition $\mathfrak{g}=\mathfrak{g}_{-1} \oplus \mathfrak{g}_{0} \oplus \mathfrak{g}_{1}$ into weight spaces of $\psi$, and $\left[\mathfrak{g}_{i}, \mathfrak{g}_{j}\right] \subset \mathfrak{g}_{i+j}$. Also $\mathfrak{g}_{0}=\operatorname{Lie}(H)$, and $\hat{\mathrm{g}}=\operatorname{Lie}(\hat{G})=\mathfrak{g} \oplus k \cdot e$ (where $e$ is the canonical generator of Lie $(S) \cong k$ ) with $[e, x]=i x$ for $x \in \mathfrak{g}_{i}$.

(a) Suppose $G$ is reductive and splits over $k$. Then the same holds for $\hat{G}$. Choose a maximal torus $T$ of $\hat{G}$ containing $S$, let $\Phi$ be the root system of $T$ and $\hat{\mathfrak{g}}=\sum \mathrm{g}^{\alpha}$ the root space decomposition. Then $\mathrm{g}_{i}=$ $\sum_{\alpha \in \Phi_{i}} \mathfrak{g}^{\alpha}(i= \pm 1)$ where $\Phi_{i}$ is the set of all $\alpha \in \Phi$ such that $\alpha \mid S=i$. Also $\left[\mathfrak{g}^{\alpha}, \mathfrak{g}^{\beta}\right]=0$ for $\alpha, \beta \in \Phi_{i}$ since $(\alpha+\beta) \mid S=2 i$ is not a weight of $S$ in $g$. It follows that the root subgroups $U^{\alpha}, U^{\beta}$ corresponding to $\mathrm{g}^{\alpha}, \mathrm{g}^{\beta}$ commute, and $U^{ \pm}=\prod_{a \in \Phi_{ \pm 1}} U^{\alpha}$ is a vector group, isomorphic with the additive group of $\mathfrak{g}_{ \pm 1}$. The exponential maps $\exp _{\alpha}: \mathfrak{g}^{\alpha} \rightarrow U^{\alpha}$ satisfy $\psi_{t} \cdot \exp _{\alpha}(x)=$ $\operatorname{Int}(s) \cdot \exp _{\alpha}(x)=\exp _{\alpha}(\operatorname{Ad} s \cdot x)=\exp _{\alpha}(t x)$ for all $t \in k_{m}$, where we set $s=$ $(1, t) \in S . \quad$ Now $\hat{\Omega}=U^{-} \cdot H \cdot S \cdot U^{+}$contains the big cell of $\hat{G}$ defined by an ordering of $\Phi$ with the property that $\Phi_{1}$ consists of positive roots. Hence $\hat{\Omega}$ is open and dense in $\hat{G}$, and therefore $U^{-} \cdot H \cdot U^{+}=\hat{\Omega} \cap G$ is open and dense in $G$. By the remark in 3.2, $\psi$ is an elementary action.

(b) If $G$ is reductive but not split over $k$ it splits over an fppf extension $K$ of $k$. Thus we have subgroups $\tilde{U}^{ \pm}$of $G_{K}$ such that $H_{K}, \tilde{U}^{ \pm}$satisfy the conditions of 3.1, and we have to show that $\tilde{U}^{ \pm}$is defined over $k$. By faithfully flat descent, it suffices to show that

$$
\tilde{U}^{ \pm} \otimes_{i_{1}}\left(K \otimes_{k} K\right)=\tilde{U}^{ \pm} \otimes_{i_{2}}\left(K \otimes_{k} K\right)
$$

where $i_{1}, i_{2}: K \rightarrow K \otimes_{k} K$ are the maps $a \mapsto a \otimes 1$ and $a \mapsto 1 \otimes a$. Since $\psi$ is defined over $k$ the two base extensions of $\psi_{K}$ induced by $i_{1}$ and $i_{2}$ are the same, and thus (1) follows from 3.6(a).

(c) Let $k$ be a field of characteristic 0 . Then $g_{ \pm 1}$ is an algebraic Lie algebra since it is the derived algebra of $k \cdot e \oplus \mathfrak{g}_{ \pm 1}([5$, p. 262, 2.6]). For 
$x \in \mathfrak{g}_{ \pm 1}$ we have $\left(\operatorname{ad}_{\hat{g}}(x)\right)^{3}=0$ and $\operatorname{ad} x \cdot e=-[e, x]=\mp x$.

Hence $x$ is nilpotent. Therefore $\mathfrak{g}_{ \pm 1}$ is the Lie algebra of a unique subgroup $U^{ \pm}$of $G$ which is isomorphic with the additive group of $\mathfrak{g}_{ \pm 1}$ under exp. Now it follows easily that $H, U^{ \pm}$satisfy the conditions of 3.1.

\section{§4. The Jordan pair associated with an elementary action}

The notations of $\S \S 2,3$ will be used throughout.

4.1. Theorem. Let $\psi$ be an elementary action. There exists a unique Jordan pair structure on the pair $\mathfrak{B}=\left(\mathfrak{B}^{+}, \mathfrak{B}^{-}\right)$of $k$-modules, and a unique morphism $b: W \rightarrow H$ with the following property: For all $(x, y) \in \mathfrak{B}_{R}^{+} \times \mathfrak{B}_{R}^{-}$ $(R \in k$-alg) we have $\exp (x) \exp (y) \in \Omega(R) \Leftrightarrow(x, y)$ quasi-invertible and in this case,

$$
\exp (x) \exp (y)=\exp \left(y^{x}\right) b(x, y) \exp \left(x^{y}\right) .
$$

(b) $H$ acts on $\mathfrak{B}$ by automorphisms via the adjoint representation (cf. $3.6(b))$.

(c) The morphism b satisfies

$$
\begin{gathered}
\operatorname{Ad} b(x, y) \cdot z=B(x, y) z, \quad \operatorname{Ad} b(x, y) \cdot w=B(y, x)^{-1} w, \\
h b(x, y) h^{-1}=b(\operatorname{Ad} h \cdot x, \operatorname{Ad} h \cdot y), \\
b\left(t x, t^{-1} y\right)=b(x, y), \\
b(x, y) b\left(x^{y}, w\right)=b(x, y+w), \\
b\left(z, y^{x}\right) b(x, y)=b(x+z, y),
\end{gathered}
$$

for all $t \in k_{m},(x, y) \in W, h \in H,(z, w) \in \mathfrak{B}_{a}^{+} \times \mathfrak{B}_{a}^{-}$such that $(x, y+w)$ and $(x+z, y)$ are quasi-invertible.

In the examples of 3.2 one checks easily that the associated Jordan pair is isomorphic with the Jordan pair of rectangular (alternating, symmetric) matrices with quadratic maps $Q(x) y=x \cdot{ }^{t} y \cdot x$.

4.2. Let $X \subset \mathfrak{B}_{a}^{+} \times \mathfrak{B}_{a}^{-}$be the inverse image of $\Omega$ under the map $(x, y)$ $\mapsto \exp (x) \exp (y)$. Then $X$ is open and dense. By 3.4, we can write

$$
\exp (x) \exp (y)=\exp \left(f_{-}(x, y)\right) b(x, y) \exp \left(f_{+}(x, y)\right)
$$

for all $(x, y) \in X$, with unique morphisms $f_{ \pm}: X \rightarrow \mathfrak{B}_{a}^{ \pm}$and $b: X \rightarrow H$. By applying $\psi_{t}$ to (1) and comparing terms in $U^{-}, H, U^{+}$we get that $(x, y) \in X$ if and only if $\left(t x, t^{-1} y\right) \in X$ and then 


$$
\begin{gathered}
b\left(t x, t^{-1} y\right)=b(x, y), \\
t^{-1} f_{-}(x, y)=f_{-}\left(t x, t^{-1} y\right), \quad t f_{+}(x, y)=f_{+}\left(t x, t^{-1} y\right) .
\end{gathered}
$$

Consider now the elementary action $\psi^{\prime}=\psi^{-1}$ (cf. 3.1). Then the formula analogous to (1) is

$$
\exp (y) \exp (x)=\exp \left(f_{-}^{\prime}(y, x)\right) b^{\prime}(y, x) \exp \left(f_{+}^{\prime}(y, x)\right) .
$$

By taking inverses in (1) and using (2) and (3) for $t=-1$ we get

$$
\exp (y) \exp (x)=\exp \left(f_{+}(x, y)\right) b(x, y)^{-1} \exp \left(f_{-}(x, y)\right) \text {. }
$$

Comparison with (4) yields $f_{\mp}^{\prime}(y, x)=f_{ \pm}(x, y)$ and

$$
b^{\prime}(y, x)=b(x, y)^{-1} \text {. }
$$

To shorten notation, write

$$
f_{+}(x, y)=f_{-}^{\prime}(y, x)=x^{y}, \quad f_{-}(x, y)=f_{+}^{\prime}(y, x)=y^{x} .
$$

Now (1) and (3) read

$$
\begin{gathered}
\exp (x) \exp (y)=\exp \left(y^{x}\right) b(x, y) \exp \left(x^{y}\right), \\
t\left(x^{y}\right)=(t x)^{t-1} y, \quad t^{-1}\left(y^{x}\right)=\left(t^{-1} y\right)^{t x} .
\end{gathered}
$$

If we apply Int $(h)$ to (8), use $3.6(b)$ and compare terms we get 4.1 .2 and

$$
\operatorname{Ad} h \cdot\left(x^{y}\right)=(\operatorname{Ad} h \cdot x)^{\operatorname{Ad} h \cdot y} .
$$

4.3. Lemma. Let $(x, y) \in X,(z, w) \in \mathfrak{B}_{a}^{+} \times \mathfrak{B}_{a}^{-} . \quad$ Then $(x+z, y) \in X$ if and only if $\left(z, y^{x}\right) \in X$, and then

$$
\begin{gathered}
(x+z)^{y}=x^{y}+\operatorname{Ad} b(x, y)^{-1} \cdot z^{\left(y^{x}\right)}, \\
y^{x+z}=\left(y^{x}\right)^{z}, \\
b\left(z, y^{x}\right) b(x, y)=b(x+z, y) .
\end{gathered}
$$

Similarly, $(x, y+w) \in X$ if and only if $\left(x^{y}, w\right) \in X$, and then

$$
\begin{gathered}
(y+w)^{x}=y^{x}+\operatorname{Ad} b(x, y) \cdot w^{(x y)}, \\
x^{y+w}=\left(x^{y}\right)^{w}, \\
b(x, y+w)=b(x, y) b\left(x^{y}, w\right) .
\end{gathered}
$$

Proof. We have $\exp (x+z)=\exp (z) \exp \left(y^{x}\right) b(x, y) \exp \left(x^{y}\right)$. This shows that $(x+z, y) \in X$ if and only if $\left(z, y^{x}\right) \in X$. Assuming this to be the case, 
we get, by 3.6 ,

$$
\begin{aligned}
\exp & (x+z) \exp (y) \\
& =\exp \left(y^{x+z}\right) b(x+z, y) \exp \left((x+z)^{y}\right) \\
& =\exp \left(\left(y^{x}\right)^{z}\right) b\left(z, y^{x}\right) \exp \left(z^{\left(y^{x}\right)}\right) b(x, y) \exp \left(x^{y}\right) \\
& =\exp \left(\left(y^{x}\right)^{z}\right) b\left(z, y^{x}\right) b(x, y) \exp \left(\operatorname{Ad} b(x, y)^{-1} \cdot z^{\left(y^{x}\right)}+x^{y}\right),
\end{aligned}
$$

and comparing terms in $U^{-}, H, U^{+}$yields (1)-(3). Now (4)-(6) follow from this by passing to $\left(G, \psi^{-1}\right)$, in view of 4.2 .

4.4. Lemma. Let $(x, y) \in \mathfrak{B}_{R}^{+} \times \mathfrak{B}_{\bar{R}}^{-}, t \in R, R \in k$-alg. Then $(t x, y) \in X(R)$ if and only if $(x, t y) \in X(R)$, and in this case,

$$
\begin{gathered}
b(t x, y)=b(x, t y), \\
(t x)^{y}=t\left(x^{t y}\right), \quad(t y)^{x}=t\left(y^{t x}\right) .
\end{gathered}
$$

Proof. We may assume $R=k$ after a base extension. Let $Y$ (resp. $Y^{\prime}$ ) be the inverse image of $X$ under the morphism $t \mapsto(t x, y)$ (resp. $t \mapsto(x, t y)$ ) from $k_{a}$ to $\mathfrak{B}_{\alpha}^{+} \times \mathfrak{B}_{a}^{-}$. Then $Y$ and $Y^{\prime}$ are open subschemes of $k_{a}$. To show that they are equal it suffices to show that $Y(K)=Y^{\prime}(K)$ for all fields $K \in k$-alg. Thus we have to show that $(t x, y) \in X(K)$ if and only if $(x, t y) \in X(K)$, for all $t \in K$. If $t=0$ this is trivial. If $t \neq 0$ then $(x, t y) \in$ $X(K)$ if and only if $\left(t x, t^{-1} t y\right)=(t x, y) \in X(K)$ by 4.2. Now define morphisms $\varphi_{1}, \varphi_{2}: Y \rightarrow \mathfrak{B}_{a}^{+}$by $\varphi_{1}(t)=(t x)^{y}, \varphi_{2}(t)=t\left(x^{t y}\right)$. By 4.2, $\varphi_{1}$ and $\varphi_{2}$ coincide on $Y \cap k_{m}$. Since $k_{m}$ is dense in $k_{a}$ and $\mathfrak{B}_{a}^{+}$is separated we have $\varphi_{1}=\varphi_{2}$. The proof of the other two formulas is similar.

4.5. Definition of the quadratic maps $Q_{+}, Q_{-}$. Let $(x, y) \in \mathfrak{B}_{R}^{+} \times \mathfrak{B}_{R}^{-}$, $R \in k$-alg. Since $X$ is open and contains $\{0\} \times \mathfrak{B}_{a}^{-}$and $\mathfrak{B}_{a}^{+} \times\{0\}$, it follows that $(\varepsilon x, y)$ and $(x, \varepsilon y)$ are in $X(R(\varepsilon)$ ) (where $R(\varepsilon)$ is the ring of dual numbers). Thus we can write

$$
x^{\varepsilon y}=f(x, y)+\varepsilon g(x, y)
$$

with well-defined morphisms $f, g: \mathfrak{B}_{a}^{+} \times \mathfrak{B}_{a}^{-} \rightarrow \mathfrak{B}_{a}^{+}$. Since $x^{0}=x$ it follows for $\varepsilon \rightarrow 0$ that $f(x, y)=x$. We claim that $g(x, y)$ is linear in $y$ and quadratic in $x$. The first statement is clear since $g(x, y)$ is the derivative of the map $x \mapsto x^{w}$ at $w=0$ in direction $y$. By 4.4, we have

$$
\begin{aligned}
(t x)^{\varepsilon y} & =t x+\varepsilon g(t x, y)=t\left(x^{t \varepsilon y}\right) \\
& =t(x+\varepsilon g(x, t y))=t x+\varepsilon t^{2} g(x, y)
\end{aligned}
$$


for all $t \in R$, and hence $g(x, y)$ is homogeneous of degree 2 in $x$. By [19], it is quadratic in $x$. Thus there is a unique quadratic map $Q_{+}: \mathfrak{B}^{+} \rightarrow$ Hom $\left(\mathfrak{B}^{-}, \mathfrak{B}^{+}\right)$such that $g(x, y)=Q_{+}(x) \cdot y$; i.e.,

$$
x^{\varepsilon y}=x+\varepsilon Q_{+}(x) \cdot y \text {. }
$$

Similarly, we define $Q_{-}: \mathfrak{B}^{-} \rightarrow \operatorname{Hom}\left(\mathfrak{B}^{+}, \mathfrak{B}^{-}\right)$by

$$
y^{\varepsilon x}=y+\varepsilon Q_{-}(y) \cdot x \text {. }
$$

Note that

$$
(\varepsilon x)^{y}=\varepsilon\left(x^{\varepsilon}\right)=\varepsilon x
$$

by 4.4. Also, from 4.2.10 it is clear that $H$ acts via Ad by automorphisms of $\left(Q_{+}, Q_{-}\right)$on $\left(\mathfrak{B}^{+}, \mathfrak{B}^{-}\right)$. As in 2.1, 2.2, we drop the subscripts \pm on $Q_{ \pm}$ and define $\{x y z\}$ and $B(x, y)$.

4.6. Lemma. For $(x, y),(z, w) \in \mathfrak{B}^{+} \times \mathfrak{B}^{-}, \quad h \in \operatorname{Lie}(H)$ the following formulae hold (here $[a, b]=a b a^{-1} b^{-1}$ ).

Int $(\exp x) \cdot \exp (\varepsilon y)=\exp (\varepsilon y) b(x, \varepsilon y) \exp \left(\varepsilon Q_{x} y\right)$.

$$
\begin{aligned}
& {[\exp (x), \exp (\varepsilon y)]=b(x, \varepsilon y) \exp \left(\varepsilon Q_{x} y\right),} \\
& {[\exp (y), \exp (\varepsilon z)]=\exp \left(\varepsilon Q_{y} z\right) b(\varepsilon z,-y),}
\end{aligned}
$$$$
[\exp (\varepsilon x), \exp (\varepsilon y)]=[h, \exp (\varepsilon x)]=[h, \exp (\varepsilon y)]=1,
$$$$
[\exp (z), b(x, \varepsilon y)]
$$$$
=[\exp (z),[\exp (-\varepsilon y), \exp (x)]]=\exp (\varepsilon\{x y z\}),
$$

Int $(\exp (x) \exp (y)) \cdot \exp (\varepsilon z)$

$$
=\exp \left(\varepsilon Q_{y} z\right) b\left(x, \varepsilon Q_{y} z\right) b(\varepsilon z,-y) \exp (\varepsilon B(x, y) z) .
$$

$$
\operatorname{Ad} b(x, y) \cdot z=B(x, y) z, \quad \operatorname{Ad} b(x, y) \cdot w=B(y, x)^{-1} w .
$$

Proof. (1)-(3) are immediate from 4.2.8 and 4.5. Replacing $x$ by $\varepsilon x$ in (2) we get the first formula of (4) since $b(\varepsilon x, \varepsilon y)=b\left(\varepsilon^{2} x, y\right)=b(0, y)=1$ 4.4. By 3.6, $[h, \exp (\varepsilon x)]=\exp (\varepsilon \operatorname{Ad} h \cdot x-\varepsilon x)$. Since $h \in \operatorname{Lie}(H)$, Ad $h \cdot x$ is of the form $x+\varepsilon x^{\prime}$ and hence $\varepsilon \operatorname{Ad} h \cdot x=\varepsilon x+\varepsilon^{2} x^{\prime}=\varepsilon x$. Similarly, one proves the third formula. To prove (5), we use (1), (4), 4.5, and 4.3.3:

$$
\begin{aligned}
& \operatorname{Int}(\exp (z)) \cdot b(x, \varepsilon y) \\
& \quad=\operatorname{Int}(\exp (z)) \cdot\left(\exp (-\varepsilon y) \exp (x) \cdot \exp (\varepsilon y) \exp \left(-x^{\varepsilon y}\right)\right)
\end{aligned}
$$




$$
\begin{aligned}
& =\exp (z) \exp (-\varepsilon y) \exp (x) \exp (\varepsilon y) \exp \left(-x^{\varepsilon y}-z\right) \\
& =\exp (-\varepsilon y) b(z,-\varepsilon y) \exp \left(x+z-\varepsilon Q_{z} y\right) \exp (\varepsilon y) \exp \left(-x-z-\varepsilon Q_{x} y\right) \\
& =\exp (-\varepsilon y) b(z,-\varepsilon y) \operatorname{Int}(\exp (x+z)) \cdot\left(\exp (\varepsilon y) \exp \left(-\varepsilon Q_{x} y-\varepsilon Q_{z} y\right)\right) \\
& =\exp (-\varepsilon y) b(z,-\varepsilon y) \exp (\varepsilon y) b(x+z, \varepsilon y) \exp \left(\varepsilon\left(Q_{x+z} y-Q_{x} y-Q_{z} y\right)\right) \\
& =b(-z, \varepsilon y) b(x+z, \varepsilon y) \exp (\varepsilon\{x y z\})=b(x, \varepsilon y) \cdot \exp (\varepsilon\{x y z\}) .
\end{aligned}
$$

Now (5) follows from (1), (2) and (4). For (6) we use the commutator formula $[a b, c]=(\operatorname{Int}(a) \cdot[b, c])[a, c]$, for $a=\exp (x), b=\exp (y), c=\exp (\varepsilon z)$. Then $[a, c]=1$ and hence

$$
\begin{aligned}
{[a b, c] } & =\operatorname{Int}(a) \cdot[b, c]=\operatorname{Int}(a) \cdot\left(\exp \left(\varepsilon Q_{y} z\right) b(\varepsilon z,-y)\right) \\
= & \exp \left(\varepsilon Q_{y} z\right) b\left(x, \varepsilon Q_{y} z\right) \exp \left(\varepsilon Q_{x} Q_{y} z\right) \exp (-\varepsilon\{x y z\}) b(\varepsilon z,-y) \\
= & \exp \left(\varepsilon Q_{y} z\right) b\left(x, \varepsilon Q_{y} z\right) b(\varepsilon z,-y) \exp \left(-\varepsilon\{x y z\}+\varepsilon Q_{x} Q_{y} z\right) .
\end{aligned}
$$

Multiplying by $\exp (\varepsilon z)$ on the right we get (6). Finally, if $(x, y) \in X$ then

$$
\begin{aligned}
& \text { Int }(\exp (x) \exp (y)) \cdot \exp (\varepsilon z) \\
& \quad=\operatorname{Int}\left(\exp \left(y^{x}\right) b(x, y) \exp \left(x^{y}\right)\right) \cdot \exp (\varepsilon z) \\
& \quad=\operatorname{Int}\left(\exp \left(y^{x}\right)\right) \operatorname{Int}(b(x, y)) \cdot \exp (\varepsilon z) \\
& \quad=\exp \left(y^{x}\right) \exp (\varepsilon \operatorname{Ad} b(x, y) \cdot z) \exp \left(-y^{x}\right) \\
& \quad=\exp (u) \cdot h \cdot \exp (\varepsilon \operatorname{Ad} b(x, y) \cdot z)
\end{aligned}
$$

where the exact form of $u \in \mathfrak{B}^{-}$and $h \in H$ is not important. Comparing terms in $U^{+}$with (6) we get the first formula of (7), and the second one follows by passing to $\psi^{-1}$.

4.7. Lemma. If $(x, y) \in X$ then $B(x, y)$ and $B(x, y)$ are invertible, and

$$
x^{y}=B(x, y)^{-1}\left(x-Q_{x} y\right), \quad y^{x}=B(y, x)^{-1}\left(y-Q_{y} x\right) .
$$

Proof. By 4.6.7, $B(x, y)$ is invertible. By 4.5.3, 4.3.1, 4.6.7,

$$
((1+\varepsilon) x)^{y}=(x+\varepsilon x)^{y}=x^{y}+\varepsilon B(x, y)^{-1} x .
$$

On the other hand, by $4.4,4.3$,

$$
\begin{aligned}
((1+\varepsilon) x)^{y}=(1+\varepsilon)\left(x^{(1+\varepsilon) y}\right) & =(1+\varepsilon)\left(\left(x^{\varepsilon y}\right)^{y}\right) \\
=(1+\varepsilon)\left(x+\varepsilon Q_{x} y\right)^{y} & =(1+\varepsilon)\left(x^{y}+\varepsilon B(x, y)^{-1} Q_{x} y\right) .
\end{aligned}
$$

Comparing coefficients at $\varepsilon$ we get the first formula, and the second one follows by passing to $\psi^{-1}$.

4.8. Lemma. The quadratic maps $Q_{+}, Q_{-}$satisfy the Jordan identities. 
Proof. From 4.5.4 and 4.6.7, $B(x, y) Q_{z}=Q(B(x, y) z) B(y, x)^{-1}$ for all $(x, y) \in X, z \in \mathfrak{B}_{a}^{+}$, and since $X$ is dense this implies

$$
B(x, y) Q_{z} B(y, x)=Q(B(x, y) z)
$$

for all $x, z \in \mathfrak{B}_{a}^{+}, y \in \mathfrak{B}_{a}^{-}$. By expanding and comparing terms of equal degree we get (among other identities)

$$
D(x, y) Q_{z}+Q_{z} D(y, x)=Q(z,\{x y z\}) .
$$

Now compute $x^{y+\varepsilon w}$ for $(x, y) \in X$ in two ways, using 4.3:

$$
\begin{aligned}
x^{x+\varepsilon w} & =\left(x^{y}\right)^{\varepsilon w}=x^{y}+\varepsilon Q\left(x^{y}\right) \cdot w \\
& =\left(x^{\varepsilon w}\right)^{y}=\left(x+\varepsilon Q_{x} w\right)^{y}=x^{y}+\varepsilon B(x, y)^{-1} \cdot Q_{x} w .
\end{aligned}
$$

By (1) and 4.7, this implies $Q_{x}=B(x, y) Q\left(x^{y}\right)=Q\left(x-Q_{x} y\right) B(y, x)^{-1}$, and therefore

$$
Q\left(x-Q_{x} y\right)=Q_{x} B(y, x) .
$$

Again by density of $X$, this holds for all $(x, y) \in \mathfrak{B}_{a}^{+} \times \mathfrak{B}_{a}^{-}$. By expanding and comparing terms of equal degree we get

$$
\begin{gathered}
Q\left(x, Q_{x} y\right)=Q_{x} D(y, x), \\
Q\left(Q_{x} y\right)=Q_{x} Q_{y} Q_{x} .
\end{gathered}
$$

Setting $z=x$ in (2) and comparing with (4) we have, since $\{x y x\}=2 Q_{x} y$,

$$
D(x, y) Q_{x}=Q_{x} D(y, x) .
$$

Let $(x, y) \in X$. Then by 4.3 and 4.6.7, $B(x,-\varepsilon y) B\left(x^{-\varepsilon y}, y\right)=B(x,(1-\varepsilon) y)$ $=B((1-\varepsilon) x, y)=B\left(x, y^{-\varepsilon y}\right) B(-\varepsilon x, y)$. By density, this holds for all $(x, y)$ $\in \mathfrak{B}_{a}^{+} \times \mathfrak{B}_{a}^{-}$. If we expand and compare terms of equal degree at $\varepsilon$ we get

$$
D\left(Q_{x} y, y\right)=D\left(x, Q_{y} x\right) .
$$

Passing to $\psi^{-1}$ it follows that (5)-(7) hold with $x$ and $y$ interchanged. This proves 4.8 .

4.9. Lemma. $H$ is closed in $G$; it is the fixed point set of $\psi$.

Proof. By definition, $H$ is contained in the fixed point set, say $H^{\prime}$, of $\psi$. Conversely let $g \in H^{\prime}(k)$. After an fppf extension we may assume that $g=\exp (x) \exp (y) h \cdot \exp (z)$ with $x, z \in \mathfrak{V}^{+}, y, \in \mathfrak{B}^{-}, h \in H(k)$ (cf. 3.8). Applying $\psi_{t}$ we get $g=\exp (t x) \exp \left(t^{-1} y\right) h \cdot \exp (t z)$ for all $t \in k_{m}$. Hence 


$$
\exp ((1-t) x) \exp (y)=\exp \left(t^{-1} y\right) h \cdot \exp (t z-z) \cdot h^{-1} \in \Omega,
$$

and therefore

$$
\left.\exp ((1-t) x) \exp (y)=\exp \left(y^{(1-t) x}\right) b((1-t) x, y) \exp ([1-t) x]^{y}\right) .
$$

Comparing terms in $H, U^{+}, U^{-}$we have $b((1-t) x, y)=1$ and by 4.6 .7 and 4.7, $t^{-1} y=y^{(1-t) x}=y-Q_{y}(x-t x)$, for all $t \in k_{m}$. By comparing terms at powers of $t$ we see that $y=0$. Hence $\exp ((1-t) x)=h \cdot \exp ((t-1) z) \cdot h^{-1}$, and this implies $\exp (x)=h \cdot \exp (-z) \cdot h^{-1}$ and therefore $g=h \in H(k)$. Now by $[5$, p. $165,3.6(d)], H$ is closed in $G$.

4.10. To complete the proof of 4.1 , all that remains to be shown is that $X=W$, the subscheme of quasi-invertible pairs. By 4.7, $X \subset W$, and $x^{y}$ and $y^{x}$ are just the quasi-inverse of the Jordan pair $\mathfrak{B}$. Define a morphism $\tilde{b}: W \rightarrow G$ by

$$
\tilde{b}(x, y)=\exp \left(-y^{x}\right) \exp (x) \exp (y) \exp \left(-x^{y}\right) .
$$

Then $\tilde{b}$ extends $b$ and $X \subset \tilde{b}^{-1}(H) \subset W$. Also, $\tilde{b}^{-1}(H)$ is closed in $W$ since $H$ is closed in $G$ by 4.9. By density of $X, W=\tilde{b}^{-1}(H)$. Therefore

$$
\exp (x) \exp (y)=\exp \left(y^{x}\right) \tilde{b}(x, y) \exp \left(x^{y}\right)
$$

belongs to $\Omega$ for all $(x, y) \in W$. This proves $X=W$.

4.11. Corollary. Assume that $G$ is a scheme, and let $\mathfrak{g}$ (resp. h) denote the Lie algebra of $G$ (resp. H). Then

$$
\mathfrak{g}=\mathfrak{B}^{-} \oplus \mathfrak{h} \oplus \mathfrak{B}^{+}
$$

(direct sum of $k$-modules), and the following multiplication rules hold:

$$
[\mathfrak{h}, \mathfrak{h}] \subset \mathfrak{h}, \quad\left[\mathfrak{B}^{+}, \mathfrak{B}^{-}\right] \subset \mathfrak{h}, \quad\left[\mathfrak{h}, \mathfrak{B}^{\sigma}\right] \subset \mathfrak{B}^{\sigma}, \quad\left[\mathfrak{B}^{\sigma}, \mathfrak{B}^{\sigma}\right]=0 .
$$

For $x, z \in \mathfrak{B}^{\sigma}, y \in \mathfrak{B}^{-\sigma}, h \in \mathfrak{h}$, we have the formulas

$$
\operatorname{Ad} \exp (x) \cdot z=z,
$$$$
\operatorname{Ad} \exp (x) \cdot h=h+[x, h],
$$$$
\operatorname{Ad} \exp (x) \cdot y=y+[x, y]+Q_{x} y
$$

$$
\begin{gathered}
e^{\varepsilon[x, y]}=b(x, \varepsilon y), \\
-[z,[y, x]]=\{x y z\} .
\end{gathered}
$$

Proof. Since $\Omega$ is open in $G$ and contains the unit section, (1) 
follows from 3.4. Let $R=k\left(\varepsilon, \varepsilon^{\prime}\right)$. If we replace $x$ by $\varepsilon^{\prime} x$ in 4.6 .2 we get $e^{\varepsilon^{\prime}[x, y]}=\left[e^{\varepsilon^{\prime} x}, e^{\varepsilon y}\right]=\left[\exp \left(\varepsilon^{\prime} x\right), \exp (\varepsilon y)\right]=b\left(\varepsilon^{\prime} x, \varepsilon y\right)=b\left(x, \varepsilon \varepsilon^{\prime} y\right)$ which implies (6) in view of $\left[5\right.$, p. 210], and also shows that $\left[\mathfrak{B}^{+}, \mathfrak{B}^{-}\right] \subset \mathfrak{h}$. Now (5) and (7) follow easily from 4.6, and (4) follows from 3.6, as well as $\left[\mathfrak{h}, \mathfrak{B}^{\circ}\right] \subset \mathfrak{B}^{\sigma}$. By commutativity of $U^{o}$ we have $\left[\mathfrak{B}^{\sigma}, \mathfrak{V}^{\sigma}\right]=0$.

4.12. Corollary. Let $G_{i n} \subset G$ be as in 3.9, and let $H_{i n}$ be the subgroup sheaf of $H$ generated by $b(W)$; i.e., $H_{\text {in }}$ is the sheaf associated with the $k$-group functor $R \mapsto$ subgroup of $H(R)$ generated by all $b(x, y)$, $(x, y) \in W(R)$.

(a) The Jordan pairs associated with $(G, \psi)$ and $\left(G_{i n}, \psi\right)$ are the same.

(b) We have $H_{i n}=H \cap G_{i n}$. If $k$ is a field and $H$ is an algebraic $k$-group then $H_{i n}$ is a smooth connected algebraic k-group.

Proof. From $(*)$ of 4.1 it is clear that $(G, \psi)$ and $\left(G_{i n}, \psi\right)$ define the same Jordan pair, and that $H_{i n} \subset H \cap G_{i n}$. Let now $F=H_{i n} \cdot U^{+} \cdot U^{-} \cdot U^{+}$ $\subset G_{i n}$. We claim that $F=G_{i n}$. Let $g \in G_{i n}(R)$. Then there exists a fppf extension $S$ of $R$ such that $g=u_{1} \cdots u_{n}$ where the $u_{i}$ are alternatingly in $U^{+}(S)$ and $U^{-}(S)$. After a base extension, it therefore suffices to show the following: if $u, w \in U^{-}(k), v \in U^{+}(k)$ then there exists a fppf extension $R$ of $k$ such that $u v w \in F(R)$. Choosing $x$ as in 3.7, we have by 4.1,

$$
u v w=\left(u x^{-1}\right)(x v w) \in U^{+}(R) H_{i n}(R) U^{-}(R) U^{-}(R) H_{i n}(R) U^{+}(R) \subset F(R) .
$$

Now

$$
H \cap G_{i n}=H \cap\left(H_{i n} \cdot U^{+} \cdot U^{-} \cdot U^{+}\right)=H_{i n} \cdot\left(H \cap U^{+} U^{-} U^{+}\right)=H_{i n},
$$

by 3.6. The last assertion follows from SGA3, Exp. $\mathrm{VI}_{B}$, no. 7 .

4.13. Lemma. Let $X=\mathfrak{B}_{a}^{+} \times \mathfrak{B}_{a}^{-} \times H \times \mathfrak{B}_{a}^{+}$and $\pi: X \rightarrow G$ the epimorphism of sheaves given by $\pi(x, y, h, z)=\exp (x) \exp (y) h \cdot \exp (z)$. Then $\pi(x, y, h, z)=\pi\left(x^{\prime}, y^{\prime}, h^{\prime}, z^{\prime}\right)$ if and only if $\left(x-x^{\prime}, y\right)$ is quasi-invertible, and

$$
y^{\prime}=y^{x-x^{\prime}}, \quad h^{\prime}=b\left(x-x^{\prime}, y\right) h, \quad z^{\prime}=z+\operatorname{Ad} h^{-1} \cdot\left(x-x^{\prime}\right)^{y} .
$$

This follows easily from 4.1 and 3.6.

4.14. Theorem (Generators and Relations). Let $(G, \psi)$ be an elementary system. Let $G^{\prime}$ be a k-group sheaf, and let $f_{0}: H \rightarrow G^{\prime}, f_{\sigma}: U^{\sigma} \rightarrow G^{\prime}(\sigma=+,-)$ be homomorphisms. Then there exist a homomorphism $f: G \rightarrow G^{\prime}$ extending $f_{0}$ and $f_{+}, f_{-}$if and only if 


$$
\begin{gathered}
f_{0}(h) f_{o}(u) f_{0}(h)^{-1}=f_{o}\left(h u h^{-1}\right), \\
f_{+}(\exp (x)) f_{-}(\exp (y))=f_{-}\left(\exp \left(y^{x}\right)\right) f_{0}(b(x, y)) f_{+}\left(\exp \left(x^{y}\right)\right),
\end{gathered}
$$

for all $h \in H, u \in U^{\sigma},(x, y) \in W$. (Since $G$ is generated by $H, U^{+}$, and $U^{-}$, such an $f$ is necessarily unique).

Proof. By 3.6 and 4.1, the conditions (1) and (2) are clearly necessary for the existence of $f$. Conversely, assume that they hold. Let $\pi: X \rightarrow G$ be as in 4.13. Let $\varphi_{\sigma}: \mathfrak{V}_{a}^{\sigma} \rightarrow G^{\prime}$ be given by $\varphi_{\sigma}=f_{\sigma} \circ \exp$ and $\varphi: X \rightarrow G^{\prime}$ by $\varphi(x, y, h, z)=\varphi_{+}(x) \varphi_{-}(y) f_{0}(h) \varphi_{+}(z)$. If $f$ exists then we have $f \circ \pi=\varphi$. To prove existence, we use the fact that the diagram

$$
X \times{ }_{G} X \underset{\mathrm{pr}_{2}}{\stackrel{\mathrm{pr}_{1}}{\longrightarrow}} X \stackrel{\pi}{\longrightarrow} G
$$

of $k$-sheaves is exact (cf. [5, p. 292]). Therefore it suffices to show that $\pi(w)=\pi\left(w^{\prime}\right)$ implies $\varphi(w)=\varphi\left(w^{\prime}\right)$, for all $w, w^{\prime} \in X$. Thus let $w=(x, y, h, z)$ and $w^{\prime}=\left(x^{\prime}, y^{\prime}, h^{\prime}, z^{\prime}\right)$ and assume that $\pi(w)=\pi\left(w^{\prime}\right)$. Вy (1) and (2) and 4.13 we then get

$$
\begin{aligned}
\varphi_{+}\left(x-x^{\prime}\right) \varphi_{-}(y) & =\varphi_{-}\left(y^{x-x^{\prime}}\right) f_{0}\left(b\left(x-x^{\prime}, y\right)\right) \varphi_{+}\left(\left(x-x^{\prime}\right)^{y}\right) \\
& =\varphi_{-}\left(y^{\prime}\right) f_{0}\left(h^{\prime} h^{-1}\right) \varphi_{+}\left(\operatorname{Ad} h \cdot\left(z^{\prime}-z\right)\right) \\
& =\varphi_{-}\left(y^{\prime}\right) f_{0}\left(h^{\prime}\right) \varphi_{+}\left(z^{\prime}-z\right) f_{0}(h)^{-1}
\end{aligned}
$$

which implies $\varphi(w)=\varphi\left(w^{\prime}\right)$. This proves the existence of $f: G \rightarrow G^{\prime}$ satisfying $f \circ \pi=\varphi$. We still have to show that $f$ is multiplicative. If $v \in U^{+}$, $g \in G$ then

$$
f(g v)=f(g) f(v), \quad f(v g)=f(v) f(g) .
$$

Indeed, we may assume, by passing if necessary to a fppf base extension, that $g=\exp (x) \exp (y) h \cdot \exp (z), v=\exp \left(z^{\prime}\right)$, and then

$$
\begin{aligned}
f(g v) & =f\left(\exp (x) \exp (y) h \cdot \exp \left(z+z^{\prime}\right)\right) \\
& =\varphi_{+}(x) \varphi_{-}(y) f_{0}(h) \varphi_{+}\left(z+z^{\prime}\right) \\
& =\varphi_{+}(x) \varphi_{-}(y) f_{0}(h) \varphi_{+}(z) \varphi_{+}\left(z^{\prime}\right)=f(g) f(v) .
\end{aligned}
$$

Similarly, one proves the second formula, and also

$$
f(h g)=f(h) f(g), \quad f(g h)=f(g) f(h),
$$

for $g \in G, h \in H$. Finally, we show that 


$$
f(u v w)=f(u) f(v) f(w),
$$

for $u, w \in U^{-}, v \in U^{+}$. Possibly after passing to an fppf base extension, there exists an element $x \in U^{+}$such that $u x^{-1} \in \Omega^{-1}$ and $x v w \in \Omega$ (see 3.7). Let $u x^{-1}=z h y, x v w=y^{\prime} h^{\prime} z^{\prime}$ where $z, z^{\prime} \in U^{+}, h, h^{\prime} \in H, y, y^{\prime} \in U^{-}$. Then we have by (3) and (4) that

$$
\begin{aligned}
f(u v w) & =f\left(u x^{-1} \cdot x v w\right)=f\left(z h y \cdot y^{\prime} h^{\prime} z^{\prime}\right) \\
& =f(z) f(h) f(y) f\left(y^{\prime}\right) f\left(h^{\prime}\right) f\left(z^{\prime}\right)=f(z h y) f\left(y^{\prime} h^{\prime} z^{\prime}\right) \\
& =f\left(u x^{-1}\right) f(x v w)=f(u) f(x)^{-1} f(x) f(v) f(w) \\
& =f(u) f(v) f(w) .
\end{aligned}
$$

Now the multiplicativity of $f$ is an easy consequence of (3)-(5), in view of the fact that $G$ is generated (as a $k$-group sheaf) by $H, U^{+}$, and $U^{-}$.

4.15. Corollary. Let $(G, \psi)$ and $\left(G^{\prime}, \psi^{\prime}\right)$ be elementary systems with associated Jordan pairs $\mathfrak{B}$ and $\mathfrak{B}^{\prime}$.

(a) Let $f:(G, \psi) \rightarrow\left(G^{\prime}, \psi^{\prime}\right)$ be a homomorphism, let

$$
f_{0}=f \mid H: H \rightarrow H^{\prime},
$$

and define $\varphi_{a}: \mathfrak{B}_{a}^{\sigma} \rightarrow \mathfrak{B}_{a}^{\prime}$ by

$$
f \circ \exp =\exp ^{\prime} \circ \varphi_{\sigma} \quad(\text { cf. 3.5) }
$$

Then $\left(\varphi_{+}, \varphi_{-}\right): \mathfrak{B} \rightarrow \mathfrak{B}^{\prime}$ is a homomorphism of Jordan pairs, and we have

$$
\begin{gathered}
\operatorname{Ad} f_{0}(h) \cdot \varphi_{o}(z)=\varphi_{o}(\operatorname{Ad} h \cdot z), \\
f_{0}(b(x, y))=b^{\prime}\left(\varphi_{+}(x), \varphi_{-}(y)\right),
\end{gathered}
$$

for all $z \in \mathfrak{B}_{a}^{a},(x, y) \in W, \sigma= \pm$.

(b) Conversely, let $f_{0}: H \rightarrow H^{\prime}$ be a homomorphism, let $\left(\varphi_{+}, \varphi_{-}\right): \mathfrak{B} \rightarrow \mathfrak{B}^{\prime}$ be a homomorphism of Jordan pairs, and assume that (3) and (4) hold. Then there exists a unique homomorphism $f:(G, \psi) \rightarrow\left(G^{\prime}, \psi^{\prime}\right)$ such that (1) and (2) hold.

Proof. (a) By 4.9, $f$ maps $H$ into $H^{\prime}$. Thus (3) follows from 3.6. If we apply $f$ to $(*)$ of 4.1 we see that (4) holds and that $\left(\varphi_{+}, \varphi_{-}\right)$preserves quasi-inverses. By 4.5 , it follows that $\left(\varphi_{+}, \varphi_{-}\right)$is a homomorphism of Jordan pairs.

(b) Define $f_{\sigma}: U^{\sigma} \rightarrow G^{\prime}$ by (2). Then one checks that $f_{0}, f_{ \pm}$satisfy the conditions of 4.14 and hence extend to a homomorphism $f: G \rightarrow G^{\prime}$ which is easily seen to be compatible with $\psi$ and $\psi^{\prime}$. 


\section{§5. The Existence theorem and applications}

5.1. We consider the question of how to reconstruct $G$, given $H$ and the Jordan pair $\mathfrak{B}$, and introduce the following concept. Let $\mathfrak{B}$ be a Jordan pair over $k$, let $H$ be a separated $k$-group sheaf, and let $\rho: H \rightarrow$ Aut (i) be a homomorphism. Also let $b: W \rightarrow H$ be a morphism from the subscheme of quasi-invertible pairs of $\mathfrak{B}_{a}^{+} \times \mathfrak{B}_{a}^{-}$into $H$. Then the quadruple $\mathscr{J}=(\mathfrak{B}, H, \rho, b)$ is called a Jordan system if the following identities hold for $(x, y) \in W,(z, w) \in \mathfrak{B}_{a}^{+} \times \mathfrak{B}_{a}^{-}, h \in H, t \in k_{m}$.

$$
\begin{gathered}
\rho(b(x, y))=\beta(x, y), \\
h b(x, y) h^{-1}=b\left(h_{+}(x), h_{-}(y)\right), \\
b\left(t x, t^{-1} y\right)=b(x, y), \\
b(x, y) b\left(x^{y}, w\right)=b(x, y+w), \\
b\left(z, y^{x}\right) b(x, y)=b(x+z, y) .
\end{gathered}
$$

Here $\beta$ is as in 2.2 and $\rho(h)=\left(h_{+}, h_{-}\right)$.

A homomorphism of Jordan systems, $\Phi: \mathscr{J} \rightarrow \mathscr{J}^{\prime}$, is a pair $\Phi=\left(f_{0}, \varphi\right)$ where $f_{0}: H \rightarrow H^{\prime}$ is a group homomorphism, and $\varphi=\left(\varphi_{+}, \varphi_{-}\right): \mathfrak{B} \rightarrow \mathfrak{V}^{\prime}$ is a Jordan pair homomorphism, such that

$$
\begin{gathered}
\rho^{\prime}\left(f_{0}(h)\right) \circ \varphi=\varphi \circ \rho(h), \\
f_{0}(b(x, y))=b^{\prime}\left(\varphi_{+}(x), \varphi_{-}(y)\right),
\end{gathered}
$$

for all $h \in H,(x, y) \in W$. From 4.1 it is clear that every elementary system $(G, \psi)$ defines a Jordan system $J(G, \psi)=(\mathfrak{B}, H, \rho, b)$ where $\rho$ is given by the adjoint representation of $H$ on $\mathfrak{B}^{+}$and $\mathfrak{B}^{-}$. Now 4.15 shows that $J$ is a covariant functor from the category of elementary systems to the category of Jordan systems which is fully faithful. In fact, $J$ is an equivalence of categories since we have

5.2. THEOREM. For every Jordan system $\mathscr{J}$ there exists an elementary system $(G, \psi)$ whose associated Jordan system is $\mathscr{J}$.

The idea of the proof is simple: let $\mathscr{J}=(\mathfrak{B}, H, \rho, b)$ and let $X=\mathfrak{B}_{a}^{+}$ $\times \mathfrak{B}_{a}^{-} \times H \times \mathfrak{B}_{a}^{+}$. If $G$ exists then it is the quotient of $X$ by the equivalence relation given in 4.13. Thus we take this as the definition of $G$ and show, rather laboriously, that it has the required properties.

Define then a subfunctor $E \subset X \times X$ as follows. For $R \in k$-alg, $E(R)$ 
is the set of all $\left((x, y, h, z),\left(x^{\prime}, y^{\prime}, h^{\prime}, z^{\prime}\right)\right)$ in $X(R) \times X(R)$ for which $\left(x-x^{\prime}, y\right)$ is quasi-invertible, and

$$
y^{\prime}=y^{x-x^{\prime}}, \quad h^{\prime}=b\left(x-x^{\prime}, y\right) h, \quad h_{+}\left(z^{\prime}-z\right)=\left(x-x^{\prime}\right)^{y} .
$$

By 2.2, $E$ may also be described by the equations

$$
\begin{aligned}
& B\left(y, x-x^{\prime}\right) y^{\prime}=y-Q_{y}\left(x-x^{\prime}\right), \\
& B\left(y, x-x^{\prime}\right) Q_{y^{\prime}}\left(x-x^{\prime}\right)=Q_{y}\left(x-x^{\prime}\right), \\
& h^{\prime}=b\left(x-x^{\prime}, y\right) h, \\
& B\left(x-x^{\prime}, y\right) h_{+}\left(z^{\prime}-z\right)=x-x^{\prime}-Q_{x-x^{\prime}} \cdot y,
\end{aligned}
$$

with values in separated $k$-functors, and is therefore closed in $X \times X$.

In the following lemmas, we will use standard properties of the quasiinverse in a Jordan pair without comment; in particular the formulas $x^{y+w}=\left(x^{y}\right)^{w},(x+z)^{y}=x^{y}+B(x, y)^{-1} \cdot z^{\left(y^{x}\right)}, B(x, y) B\left(x^{y}, w\right)=B(x, y+w)$, $B\left(z, y^{x}\right) B(x, y)=B(x+z, y)$. See [15, §3].

\subsection{Lemma. $E$ is an equivalence relation on $X$.}

Proof. We have to show that $E(R)$ is (the graph of) an equivalence relation on $X(R)$, for all $R \in k$-alg. Since $y^{0}=y, 0^{y}=0, b(0, y)=1$ (which follows from 5.1.1 for $z=x=0$ ) we have reflexivity. Now assume that $(* *)$ holds. Then $\left(x^{\prime}-x, y\right)$ is quasi-invertible and $y=\left(y^{\prime}\right)^{x^{\prime}-x}$,

$$
h=b\left(x-x^{\prime}, y\right)^{-1} h^{\prime}=b\left(-\left(x-x^{\prime}\right), y^{x-x^{\prime}}\right) h^{\prime}=b\left(x^{\prime}-x, y^{\prime}\right) h^{\prime}
$$

by 5.1 .5 , and by 5.1 .1 ,

$$
\begin{aligned}
h_{+}^{\prime}\left(z-z^{\prime}\right) & =B\left(x-x^{\prime}, y\right) h_{+}\left(z-z^{\prime}\right)=-B\left(x-x^{\prime}, y\right)\left(x-x^{\prime}\right)^{y} \\
& =-\left(x-x^{\prime}-Q\left(x-x^{\prime}\right) y\right)=\left(x^{\prime}-x\right)+Q\left(x^{\prime}-x\right) y \\
& =x^{\prime}-x+Q\left(x^{\prime}-x\right)\left(y^{\prime}\right)^{x^{\prime}-x}=\left(x^{\prime}-x\right)^{y^{\prime}} .
\end{aligned}
$$

This shows that $E$ is symmetric. To prove transitivity, assume (**) and also that $\left(x^{\prime}-x^{\prime \prime}, y^{\prime}\right)$ is quasi-invertible and

$$
y^{\prime \prime}=\left(y^{\prime}\right)^{x^{\prime}-x^{\prime \prime}}, \quad h^{\prime \prime}=b\left(x^{\prime}-x^{\prime \prime}, y^{\prime}\right), \quad h_{+}^{\prime}\left(z^{\prime \prime}-z^{\prime}\right)=\left(x^{\prime}-x^{\prime \prime}\right)^{y^{\prime}} .
$$

Then $\left(x-x^{\prime \prime}, y\right)$ is quasi-invertible, and

$$
y^{\prime \prime}=\left(y^{\prime}\right)^{x^{\prime}-x^{\prime \prime}}=\left(y^{x-x^{\prime}}\right)^{x^{\prime}-x^{\prime \prime}}=y^{x-x^{\prime}+x^{\prime}-x^{\prime \prime}}=y^{x-x^{\prime \prime}} .
$$

Also, 


$$
\begin{aligned}
h^{\prime \prime} & =b\left(x^{\prime}-x^{\prime \prime}, y^{\prime}\right) b\left(x-x^{\prime}, y\right) h=b\left(x^{\prime}-x^{\prime \prime}, y^{x-x^{\prime}}\right) b\left(x-x^{\prime}, y\right) h \\
& =b\left(x^{\prime}-x^{\prime \prime}+x-x^{\prime}, y\right) h=b\left(x-x^{\prime \prime}, y\right) h,
\end{aligned}
$$

by 5.1 .5 and finally,

$$
\begin{aligned}
h_{+}\left(z^{\prime \prime}-z\right) & =h_{+}\left(z^{\prime \prime}-z^{\prime}\right)+h_{+}\left(z^{\prime}-z\right) \\
& =h_{+} h_{+}^{\prime-1} h_{+}^{\prime}\left(z^{\prime \prime}-z^{\prime}\right)+\left(x-x^{\prime}\right)^{y} \\
& =B_{+}\left(x-x^{\prime}, y\right)^{-1} \cdot\left(x^{\prime}-x^{\prime \prime}\right)^{y^{\prime}}+\left(x-x^{\prime}\right)^{y} \\
& =\left(x-x^{\prime}\right)^{y}+B_{+}\left(x-x^{\prime}, y\right)^{-1} \cdot\left(\left(x^{\prime}-x^{\prime \prime}\right)^{\left(y^{\left.x-x^{\prime}\right)}\right.}\right) \\
& =\left(x-x^{\prime}+x^{\prime}-x^{\prime \prime}\right)^{y} \\
& =\left(x-x^{\prime \prime}\right)^{y}
\end{aligned}
$$

by 5.1.1.

5.4. We now define $G=X / E$ to be the quotient sheaf of $X$ by $E$, and denote by $\pi: X \rightarrow E$ the canonical map. For $(x, y, h, z) \in X(R)$, the notation

$$
\pi(x, y, h, z)=[x, y, h, z]
$$

will be used. By general properties of sheaves (cf. [5, chap. III]), we have the exact sequence of sheaves

$$
E \underset{\mathrm{pr}_{2}}{\stackrel{\mathrm{pr}_{1}}{\longrightarrow}} X \stackrel{\pi}{\longrightarrow} G \text {. }
$$

Since $E$ is closed, 1.3 shows that $G$ is separated.

5.5. Lemma. There is a well defined function $\chi$ on $G$ such that $\chi([x, y, h, z])=\operatorname{det}\left(B(x, y) \cdot h_{+}\right)$. The open subfunctor $\Omega=\chi^{-1}\left(k_{m}\right)$ of $G$ is dense and isomorphic with $\mathfrak{B}_{a}^{-} \times H \times \mathfrak{B}_{a}^{+}$under the map $i:(y, h, z) \rightarrow$ $[0, y, h, z]$.

Proof. $\chi$ is well-defined: Suppose $[x, y, h, z]=\left[x^{\prime}, y^{\prime}, h^{\prime}, z^{\prime}\right]$. Then $y^{\prime}$ $=y^{x-x^{\prime}}$ and $h_{+}^{\prime}=B\left(x-x^{\prime}, y\right) h_{+}$whence $B\left(x^{\prime}, y^{\prime}\right) h_{+}^{\prime}=B\left(x^{\prime}, y^{x-x^{\prime}}\right) B\left(x-x^{\prime}, y\right) h_{+}$ $=B(x, y) h_{+}$. Clearly $\pi^{-1}(\Omega)=W \times H \times \mathfrak{B}_{a}^{+}$which is dense in $X$. By 1.11, $\Omega$ is dense in $G$. From (**) it follows easily that $i$ is a monomorphism into $\Omega$. Conversely, if $g=[x, y, h, z] \in \Omega$ then $(x, y)$ is quasi-invertible and hence $g=\left[0, y^{x}, b(x, y) h, z+h_{+}^{-1} \cdot x^{y}\right]$ belongs to the image of $i$.

5.6. Let $\mathfrak{B}_{a}^{+}$act on $X$ on the left by addition on the first factor:

$$
u \cdot(x, y, h, z)=(u+x, y, h, z),
$$

and let $H$ act on $X$ via 


$$
f \cdot(x, y, h, z)=\left(f_{+}(x), f_{-}(y), f h, z\right) .
$$

Then these actions are compatible in the sense that

$$
f \cdot(u \cdot(x, y, h, z))=f_{+}(u) \cdot(f \cdot(x, y, h, z))
$$

and it is easily checked that they are compatible with $E$. Therefore we have actions of $\mathfrak{B}_{a}^{+}$and $H$ on $G$, compatible in the sense that $f \cdot(u \cdot g)=$ $f_{+}(u) \cdot(f \cdot g)$ for $f \in H, u \in \mathfrak{B}_{a}^{+}, g \in G$.

5.7. Lemma. There exist a unique action of $\mathfrak{B}_{a}^{-}$on $G$ on the left such that

$$
v \cdot[x, y, h, z]=\left[x^{v}, B(v, x)\left(v^{x}+y\right), b(x, v)^{-1} h, z\right]
$$

whenever $(x, y)$ is quasi-invertible. This action is compatible with the actions of $H$ and $\mathfrak{B}_{a}^{+}$in the sense that

$$
\begin{gathered}
f \cdot(v \cdot g)=f_{-}(v) \cdot(f \cdot g), \\
\left.u \cdot(v \cdot g)=v^{u} \cdot b(u, v) \cdot\left(u^{v} \cdot g\right)\right) .
\end{gathered}
$$

for all $f \in H$ and $(u, v) \in W$.

Proof. Let $v \in \mathfrak{B}^{-}$and $g \in G(k)$. After passing to an fppf extension of $k$ we may assume that $g=[x, y, h, z]$. We claim that, again after an fppf extension, we may even assume that $(x, v)$ is quasi-invertible. Indeed, let $Y_{i} \subset \mathfrak{B}_{a}^{+}$be defined by

$$
\begin{aligned}
& Y_{1}(R)=\left\{x^{\prime} \in \mathfrak{B}_{R}^{+} \mid\left(x-x^{\prime}, y\right) \text { quasi-invertible }\right\}, \\
& Y_{2}(R)=\left\{x^{\prime} \in \mathfrak{B}_{R}^{+} \mid\left(x^{\prime}, v\right) \text { quasi-invertible }\right\} .
\end{aligned}
$$

Then $Y_{1}$ and $Y_{2}$ are open and dense since $x \in Y_{1}(k)$ and $0 \in Y_{2}(k)$. Hence $Y_{1} \cap Y_{2}$ is dense, and there exists a fppf extension $R$ of $k$ such that $\left(Y_{1} \cap Y_{2}\right)(R)$ is not empty. Choosing $x^{\prime} \in Y_{1}(R) \cap Y_{2}(R)$, define $y^{\prime}, h^{\prime}, z^{\prime}$ by $(* *)$. Then $g=\left[x^{\prime}, y^{\prime}, h^{\prime}, z^{\prime}\right]$ and $\left(x^{\prime}, v\right)$ is quasi-invertible.

Now we show that (1) is well defined. Thus let $[x, y, h, z]=\left[x^{\prime}, y^{\prime}, h^{\prime}, z^{\prime}\right]$ and assume, as we may, that both $(x, v)$ and $\left(x^{\prime}, v\right)$ are quasi-invertible. By $(* *)$, we also have, setting $u=x-x^{\prime}$, that $(u, y)$ is quasi-invertible, and that

$$
y^{\prime}=y^{u}, \quad h^{\prime}=b(u, y) h, \quad h_{+}\left(z^{\prime}-z\right)=u^{y} .
$$

To shorten notation, let 


$$
f=b(x, v)^{-1}, \quad w=v^{x}, \quad s=f_{-}(w+y)=B_{-}(v, x)\left(v^{x}+y\right),
$$

and define $f^{\prime}, w^{\prime}, s^{\prime}$ analogously. We have to show: $\left(x^{v}-x^{\prime v}, s\right)$ is quasiinvertible, and

$$
\begin{gathered}
s^{\prime}=s^{\left(x^{v}-x^{\prime v}\right)}, \\
f^{\prime} h^{\prime}=b\left(x^{v}-x^{\prime v}, s\right) \cdot f \cdot h, \\
f_{+} h_{+}\left(z^{\prime}-z\right)=\left(x^{v}-x^{v}\right)^{s} .
\end{gathered}
$$

Using the formula for $(x+z)^{y}$, we have

$$
x^{v}-x^{\prime v}=B_{+}(x, v)^{-1}\left(\left(x-x^{\prime}\right)^{-v^{x}}\right)=f_{+}\left(u^{-w}\right),
$$

in particular, $(u,-w)$ is quasi-invertible. Now

$$
\left(x^{v}-x^{\prime v}, s\right)=\left(f_{+}\left(u^{-w}\right), f_{-}(y+w)\right)
$$

is quasi-invertible if and only if (since $\left(f_{+}, f_{-}\right)$is an automorphism of $\mathfrak{B}$ ) $\left(u^{-w}, y+w\right)$ is quasi-invertible, and this is the case since both $(u,-w)$ and $(u, y)$ are quasi-invertible. Also,

$$
\left(x^{v}-x^{\prime v}\right)^{s}=f_{+}\left(\left(u^{-w}\right)^{y+w}\right)=f_{+}\left(u^{y}\right)=f_{+}\left(h_{+}\left(z^{\prime}-z\right)\right),
$$

which proves (6). For (5), we have, by (2)-(5) of 5.1, that

$$
f^{\prime}=f \cdot b(u,-w)^{-1},
$$

and hence

$$
\begin{aligned}
b\left(x^{v}-x^{\prime v}, s\right) \cdot f \cdot h & =b\left(f_{+}\left(u^{-w}\right), f_{-}(y+w)\right) \cdot f \cdot h \\
& =f \cdot b\left(u^{-w}, y+w\right) f^{-1} \cdot f \cdot h=f \cdot b\left(u^{-w}, y+w\right) \cdot h \\
& =f \cdot b(u,-w)^{-1} b(u, y) \cdot h=f^{\prime} \cdot h^{\prime} .
\end{aligned}
$$

Finally,

$$
\begin{aligned}
s^{\prime} & =f_{-}^{\prime}\left(w^{\prime}+y^{\prime}\right)=f_{-}^{\prime}\left(w^{\prime}+y^{u}\right)=f_{-}^{\prime}\left(w^{-u}+y^{u}\right) \\
& =f_{-}^{\prime}\left(y^{u}-(-w)^{u}\right)=f_{-}^{\prime}\left(B_{-}(-w, u)^{-1} \cdot\left((y+w)^{(u-w)}\right)\right.
\end{aligned}
$$

and

$$
s^{\left(x^{\left.v-x^{\prime} v\right)}\right.}=\left(f_{-}(w+y)^{f+(u-w)}\right)=f_{-}\left((w+y)^{(u-w)}\right) .
$$

Now (4) follows from (7).

Next we show that (1) defines an action of $\mathfrak{B}_{a}^{-}$on $G$. Since $\Omega$ is dense in $G$ and $G$ is separated, it suffices to verify the condition $v_{1}\left(v_{2} \cdot g\right)$ 
$=\left(v_{1}+v_{2}\right) \cdot g$ for $g=[0, y, h, z]$ in $\Omega$. But then $v \cdot g=[0, v+y, h, z]$ and the assertion follows. Finally, (2) is easily verified from the definitions, and (3) needs to be checked only for $g=[0, y, h, z]$ in $\Omega$. Then we have

$$
u \cdot(v \cdot g)=u \cdot[0, v+y, h, z]=[u, v+y, h, z] .
$$

Let $d=u-Q_{u} v$. Then $\left(d, v^{u}\right)$ is quasi-invertible, and we have the formulas

$$
\begin{gathered}
b\left(d, v^{u}\right)=b(u, v), \\
d^{\left(v^{u}\right)}=u .
\end{gathered}
$$

Indeed, by (4) and (5) of 5.1 and the "symmetry principle" ([15, 3.3]),

$$
\begin{aligned}
b(u, v) & =\left(b(u, v)^{-1}\right)^{-1}=b\left(-u, v^{u}\right)^{-1}=b\left((-u)^{\left(v^{u}\right)},-v^{u}\right) \\
& =b\left(-u+Q_{u} \cdot\left(v^{u}\right)^{-u},-v^{u}\right)=b\left(d, v^{u}\right),
\end{aligned}
$$

and

$$
u^{\left(-v^{u}\right)}=u+Q_{u} \cdot\left(-v^{u}\right)^{u}=u+Q_{u} \cdot\left((-v)^{-u}\right)^{u}=d
$$

which implies (9). Now we get

$$
\begin{aligned}
v^{u} \cdot\left(b(u, v) \cdot\left(u^{v} \cdot g\right)\right) & =v^{u} \cdot\left(b(u, v) \cdot\left[u^{v}, y, h, z\right]\right) \\
& =v^{u} \cdot\left[B(u, v) \cdot u^{v}, B(v, u)^{-1} \cdot y, b(u, v) \cdot h, z\right] \\
& =v^{u} \cdot\left[d, B(v, u)^{-1} y, b(u, v) h, z\right] \\
& =\left[d^{\left(v^{u}\right)}, B(v, u)\left(v^{u}\right)^{d}+y, h, z\right] \\
& =[u, v+y, h, z]
\end{aligned}
$$

since $B(v, u)\left(v^{u+d}\right)=\left(B(v, u) \cdot v^{u}\right)^{B(u, v)-1} \cdot d=\left(v-Q_{v} u\right)^{(u v)}=v$, by (9) applied with $u$ and $v$ interchanged. This completes the proof.

5.8. LemmA. There exists a unique structure of $a$ k-group sheaf on $G$ such that

$$
[x, y, h, z] \cdot g=x \cdot(y \cdot(h \cdot(z \cdot g))),
$$

for all $(x, y, h, z) \in X, g \in G$.

Proof. By 5.6 and 5.7, the right hand side of (1) defines a map $X \times$ $G \rightarrow G$. We claim that it depends only on the equivalence class $[x, y, h, z]$. Indeed, let $[x, y, h, z]=\left[x^{\prime}, y^{\prime}, h^{\prime}, z^{\prime}\right]$. Then by $(* *), 5.6 .1$ and 5.7.3 we have, setting $u=x-x^{\prime}$, 


$$
\begin{aligned}
u \cdot(y \cdot(h \cdot(z \cdot g))) & =y^{u} \cdot\left(b(u, y) \cdot\left(u^{y} \cdot(h \cdot(z \cdot g))\right)\right) \\
& =y^{\prime} \cdot\left(h^{\prime} h^{-1} \cdot\left(h_{+}\left(z^{\prime}-z\right) \cdot(h \cdot(z \cdot g))\right)\right) \\
& =y^{\prime} \cdot\left(h^{\prime} h^{-1} \cdot\left(h \cdot\left(\left(z^{\prime}-z\right) \cdot(z \cdot g)\right)\right)\right) \\
& =y^{\prime} \cdot\left(h^{\prime} \cdot\left(z^{\prime} \cdot g\right)\right)
\end{aligned}
$$

and hence $x^{\prime} \cdot\left(y^{\prime} \cdot\left(h^{\prime} \cdot\left(z^{\prime} \cdot g\right)\right)\right)=x \cdot(y \cdot(h \cdot(z \cdot g)))$. Thus there exists a unique morphism $G \times G \rightarrow G$ satisfying (1). Clearly, $[0,0,1,0]$ is the neutral element of this multiplication. Now we prove associativity. Let $U \subset \Omega$ $\times \Omega$ be the set of all pairs $\left(g, g^{\prime}\right)=\left([0, y, h, z],\left[0, y^{\prime}, h^{\prime}, z^{\prime}\right]\right)$ for which $\left(z, y^{\prime}\right)$ is quasi-invertible. Then $U$ is open and dense in $\Omega \times \Omega$ and hence $U \times G$ is open and dense in $G \times G \times G$. Hence we only have to prove $\left(g g^{\prime}\right) g^{\prime \prime}$ $=g\left(g^{\prime} g^{\prime \prime}\right)$ for $\left(g, g^{\prime}\right) \in U$. In that case,

$$
\begin{aligned}
g g^{\prime} & =y \cdot\left(h \cdot\left(z \cdot\left[0, y^{\prime}, h^{\prime}, z^{\prime}\right]\right)\right)=y \cdot\left(h \cdot\left[z, y^{\prime}, h^{\prime}, z^{\prime}\right]\right) \\
& =y \cdot\left(h \cdot\left[0, y^{\prime z}, b\left(z, y^{\prime}\right) h^{\prime}, h_{+}^{\prime-1}\left(z^{y^{\prime}}\right)+z^{\prime}\right]\right) \\
& =\left[0, y+h_{-}\left(y^{\prime z}\right), h b\left(z, y^{\prime}\right) h^{\prime}, h_{+}^{\prime-1}\left(z^{y^{\prime}}\right)+z^{\prime}\right]=[0, v, f, w],
\end{aligned}
$$

and therefore

$$
\left(g g^{\prime}\right) g^{\prime \prime}=v \cdot\left(f \cdot\left(w \cdot g^{\prime \prime}\right)\right) .
$$

On the other hand, by 5.6 and 5.7.,

$$
\begin{aligned}
g\left(g^{\prime} g^{\prime \prime}\right) & =y \cdot\left(h \cdot\left(z \cdot\left(y^{\prime} \cdot\left(h^{\prime} \cdot\left(z^{\prime} \cdot g^{\prime \prime}\right)\right)\right)\right)\right) \\
& =y \cdot\left(h \cdot\left(y^{\prime z} \cdot\left(b\left(z, y^{\prime}\right) \cdot\left(z^{y^{\prime}} \cdot\left(h^{\prime} \cdot\left(z^{\prime} \cdot g^{\prime \prime}\right)\right)\right)\right)\right)\right) \\
& =y \cdot\left(h_{-}\left(y^{\prime 2}\right) \cdot\left(h b\left(z, y^{\prime}\right) h^{\prime} \cdot\left(h_{+}^{\prime-1}\left(z^{y^{\prime}}\right) \cdot\left(z^{\prime} \cdot g^{\prime \prime}\right)\right)\right)\right) \\
& =\left(y+h_{-}\left(y^{\prime z}\right)\right) \cdot\left(h b\left(z, y^{\prime}\right) h^{\prime} \cdot\left(\left(h_{+}^{\prime-1}\left(z^{y^{\prime}}\right)+z^{\prime}\right) \cdot g^{\prime \prime}\right)\right) \\
& =v \cdot\left(f \cdot\left(w \cdot g^{\prime \prime}\right)\right)=\left(g g^{\prime}\right) g^{\prime \prime} .
\end{aligned}
$$

Finally, it is easily checked that the inverse is given by

$$
[x, y, h, z]^{-1}=\left[-z,-h_{-}^{-1}(y), h^{-1},-x\right] .
$$

This completes our proof.

5.9. Lemma. The action $\psi$ of $k_{m}$ on $G$ defined by $\psi_{t} \cdot[x, y, h, z]=$ $\left[t x, t^{-1} y, h, t z\right]$ is elementary and the associated Jordan system is isomorphic with $\mathscr{J}=(\mathfrak{B}, H, \rho, b)$.

Proof. One shows easily that $\psi$ is a well defined action by group automorphisms. Identify $H$ with a subgroup of $G$ via $h \mapsto[0,0, h, 0]$ and define homomorphisms $f_{ \pm}: \mathfrak{V}_{a}^{ \pm} \rightarrow G$ by 


$$
f_{+}(x)=[x, 0,1,0]=[0,0,1, x], \quad f_{-}(y)=[0, y, 1,0] .
$$

Let $U^{ \pm}$be the image of $\mathfrak{B}_{a}^{ \pm}$. Then $H, U^{+}, U^{-}$satisfy (i)-(iv) of 3.1 and therefore $\psi$ is elementary. From 5.6-5.8 it follows that $H$ normalizes $U^{ \pm}$; in fact, $h f_{\sigma}(x) h^{-1}=f_{\sigma}\left(h_{\sigma} \cdot x\right)$. Finally by $(* *), f_{+}(x) f_{-}(y)=[x, y, 1,0]=$ $\left[0, y^{\prime}, h^{\prime}, z^{\prime}\right] \in \Omega$ if and only if $(x, y)$ is quasi-invertible, and then $y^{\prime}=y^{x}$, $h^{\prime}=b(x, y), z^{\prime}=x^{y}$. In view of 4.1 , this shows that the Jordan system of $(G, \psi)$ is $\mathscr{J}$ and completes the proof of 5.2.

5.10. Corollary. Let $(G, \psi)$ be an elementary system. Then there exists a unique function $\chi$ on $G$ such that $\chi(g)=\operatorname{det} B(x, y) \cdot \operatorname{Ad} h \mid \mathfrak{B}^{+}$) for $g=\exp (x) \exp (y) h \cdot \exp (z),\left(x, z \in \mathfrak{V}_{a}^{+}, y \in \mathfrak{B}_{a}^{-}, h \in H\right)$, and $\Omega$ is the open subfunctor of $G$ defined by $\chi$.

This is immediate from 5.5. Next, we discuss representability of $G$.

5.11. Proposition. Let $(G, \psi)$ be an elementary system, and assume that $G$ is a scheme. Then $H$ is a scheme, and the morphism $\pi: X \rightarrow G$ (cf. 4.13) is a finitely presented, smooth, affine, and surjective morphism of $k$-schemes. The following properties hold for $G$ if and only if they hold for $H$ :

(i) finitely presented;

(ii) locally finitely presented;

(iii) flat and locally finitely presented;

(iv) smooth.

Proof. $H$ is a scheme since it is closed in $G([5$, p. 50, 4.1]). We can factor $\pi$ as follows:

$$
X \stackrel{\cong}{\longrightarrow} U^{+} \times \Omega \stackrel{\operatorname{Id} \times \bullet}{\longrightarrow} U^{+} \times G \stackrel{\mu}{\longrightarrow} G,
$$

where the first map is the isomorphism $(x, y, h, z) \rightarrow(\exp (x), \exp (y) \cdot h \cdot \exp (z))$, $\iota: \Omega \rightarrow G$ is the inclusion, and $\mu$ is multiplication. Since $\iota$ is an open imbedding it is smooth and locally finitely presented. Let $U \subset G$ be open and affine, and let $\chi$ be as in 5.10. Then $\iota^{-1}(U)=U \cap \Omega$ is the open subscheme of $U$ defined by a single function, and is therefore itself affine. This proves that $\iota$ is affine and quasicompact (cf. [5, p. 48, 3.8, and p. 41, 2.1]) and therefore also finitely presented. It follows that $I d \times \iota$ has the same properties. The morphism $\mu: U^{+} \times G \rightarrow G$ is isomorphic with the projection $\mathrm{pr}_{2}: U^{+} \times G \rightarrow G$ (cf. [5, p. 161, 3.2]). Since $U^{+}$is finitely presented, smooth, and affine over $k, \mathrm{pr}_{2}$ and hence $\mu$ are finitely presented, smooth and affine. 
Thus we have shown that $\pi$ is finitely presented, smooth, and affine, and it is surjective since it is an epimorphism of sheaves.

If one of the properties (i)-(iv) holds for $H$ then it holds for $X$ since $\mathfrak{B}_{a}^{ \pm}$ is smooth and finitely presented. By EGA IV, 11.3.11, 17.7.5, 17.7.7, it holds for $G$. Conversely, if $G$ has one of these properties then so does $\Omega \cong H$ $\times U^{+} \times U^{-}$and therefore $H$, by faithfully flat descent $\left(U^{+} \times U^{-}\right.$being faithfully flat and finitely presented over $k$ ).

5.12. Lemma Let $(G, \psi)$ be an elementary system, and assume that $H$ is a scheme. Let $E=X \times{ }_{G} X$ be the equivalence relation defined by $\pi: X \rightarrow G$. Then $\mathrm{pr}_{1}: E \rightarrow X$ is flat and finitely presented.

Proof. From 4.13 it is clear that $E$ is isomorphic with the open subscheme $Y$ of $X \times \mathfrak{B}_{a}^{+}$defined by

$$
Y(R)=\left\{\left(x, y, h, z ; x^{\prime}\right) \in X(R) \times \mathfrak{V}_{R}^{+} \mid\left(x-x^{\prime}, y\right) \text { quasi-invertible }\right\},
$$

and that $\mathrm{pr}_{1}: E \rightarrow X$ is isomorphic with $Y \hookrightarrow X \times \mathfrak{B}_{a}^{+} \stackrel{p}{\longrightarrow} X$ where $p(x$, $\left.y, h, z ; x^{\prime}\right)=(x, y, h, z)$. Since $\mathfrak{B}_{a}^{+}$is flat and finitely presented over $k, p$ is flat and finitely presented. Also, $\mathrm{Y}$ is the open subscheme of $X \times \mathfrak{V}_{a}^{+}$ defined by the function $f\left(x, y, h, z ; x^{\prime}\right)=\operatorname{det} B\left(x-x^{\prime}, y\right)$. Hence the inclusion $Y \leftrightharpoons X \times \mathfrak{B}_{a}^{+}$is finitely presented. This finishes the proof. We remark that, in fact, $\mathrm{pr}_{1}: E \rightarrow X$ is even smooth and affine, as follows from the proof.

5.13. Proposition. Let $(G, \psi)$ be an elementary system over $k$, and assume that $k$ is noetherian and $H$ is a finitely presented $k$-scheme.

(a) $G$ is a finitely presented algebraic space (in the sense of $M$. Artin).

(b) If $k$ has Krull dimension $\leq 1$ then $G$ is a scheme.

(c) If $k$ is a Dedekind ring then $G$ is flat and affine if and only if $H$ is.

Proof. (a) and (b) follow from 5.12 and [1, Th. 3.1.1 and Th. 4B]. If $G$ is affine so is $H$ since it is closed in $G$. For the converse, we may, by $[1,2.3 .1]$, assume that $k$ is a field. Let $\hat{G}=G \rtimes k_{m}$ (semidirect) and $S=1 \times k_{m} \subset \hat{G}$. Then $S$ is contained in $\hat{G}^{0}$ and the centralizer of $S$ in $\hat{G}$ is $H \cdot S$. Hence the centre of $\hat{G}^{0}$ is contained in $H \cdot S \cong H \times k_{m}$ and is therefore affine. By [5, p. 359, 8.4], $\hat{G}$ is affine, and hence $G$ is affine.

I don't know whether $G$ is a scheme whenever $H$ is.

5.14. For any Jordan pair $\mathfrak{B}$ we can define a canonical Jordan system $\mathscr{J}(\mathfrak{B})$ by setting $H=\operatorname{Aut}(\mathfrak{B}), \rho=\mathrm{Id}$, and $b=\beta$. This follows from the 
properties of the inner automorphisms $\beta(x, y)([15, \S 3])$. The associated group is called the projective group of $\mathfrak{B}$ and is denoted by $\mathbf{P G}(\mathfrak{B})$. In the examples of $3.2, \mathbf{P G}(\mathfrak{B})$ is isomorphic with the projective group $\mathbf{P G} \mathbf{L}_{n}$ $\mathbf{P O}_{2 n}, \mathbf{P S p}_{n}$ respectively (except in the first case when $p=q=n / 2>1$ where $\mathbf{P G L}_{n}$ is an open subgroup of index 2 in $\mathbf{P G}(\mathfrak{B})$ ). As a further example, let $k$ be a field, and $\mathfrak{B}$ the Jordan pair of $1 \times 2$-matrices over a Cayley division algebra $C$ over $k$ (cf. [15, 8.15]). Then one can show that $\mathbf{P G}(\mathfrak{B})$ is an adjoint group of type $E_{6}$ whose $k$-rational points may be identified with the projective group of the Cayley plane defined by $C$ (cf. ([6]).

5.15. Proposition. Let $(G, \psi)$ be an elementary system with associated Jordan pair $\mathfrak{B}$, and let $\bar{G}=\mathbf{P G}(\mathfrak{B})$. Let $\bar{\psi}$ be the elementary action on $\bar{G}$ and $\bar{H}=\operatorname{Aut}(\mathfrak{B}), \bar{U}^{ \pm}$the corresponding subgroups.

(a) There exists a canonical homomorphism $\kappa:(G, \psi) \rightarrow(\bar{G}, \bar{\psi})$ given by $\kappa(h)=\left(\operatorname{Ad} h\left|\mathfrak{B}^{+}, \operatorname{Ad} h\right| \mathfrak{B}^{-}\right)(h \in H)$ and $k(\exp (x))=\overline{\exp }(x)\left(x \in \mathfrak{B}^{ \pm}\right)$. The kernel of $\kappa$ is the largest normal subgroup of $G$ contained in $H$; in particular, $\kappa: U^{ \pm} \rightarrow \bar{U}^{ \pm}$is an isomorphism.

(b) Let $\gamma: k_{n} \rightarrow \bar{H}$ be as in 2.3, and $\bar{S}$ the image of $\gamma$. Then $\bar{\psi}$ is given by $\bar{\psi}_{t}(g)=\gamma(t) g \gamma(t)^{-1}$, and hence $\bar{H}$ is the centralizer of $\bar{S}$ in $\bar{G}$.

(c) $\bar{H}$ contains no non-trivial normal subgroup of $\bar{G}$. The centre of $\bar{G}$ is trivial.

Proof. Define $f_{0}: H \rightarrow \bar{H}$ by $h \mapsto\left(\operatorname{Ad} h\left|\mathfrak{B}^{+}, \operatorname{Ad} h\right| \mathfrak{B}^{-}\right)$. Since the Jordan pairs of $G$ and $\bar{G}$ are the same, 4.15 (b) shows the existence of $\kappa$, and clearly $\kappa: U^{ \pm} \rightarrow \bar{U}^{ \pm}$is an isomorphism. Let $\kappa(g)=1$. After an fppf extension, we may by 3.8 assume that $g=h \cdot \exp (x) \exp (y) \exp (z)$ with $x$, $z \in \mathfrak{B}_{a}^{+}, y \in \mathfrak{B}_{a}^{-}, h \in H$. Then $k(h)^{-1}=\overline{\exp }(x) \overline{\exp }(y) \overline{\exp }(z)=1$ by $3.6(c)$. Hence $\overline{\exp }(x) \overline{\exp }(y)=\overline{\exp }(-z) \in \bar{\Omega}$ and by 4.1, $\overline{\exp }(-z)=\overline{\exp }\left(y^{x}\right) \bar{b}(x, y)$ $\overline{\exp }\left(x^{y}\right)$, which implies $y^{x}=0$, and $-z=x^{y}$. It follows that $y=0$ and $z=-x$, and therefore $g=h \in H$. Thus the kernel of $\kappa$ is contained in $H$. If $N$ is a normal subgroup of $G$ contained in $H$ then $N$ centralizes $U^{+}$and $U^{-}$since it normalizes $U^{+}$and $U^{-}$and $H \cap U^{ \pm}=\{1\}$. By 3.6, it follows that $\operatorname{Ad} h \cdot x=x$ for all $h \in N, x \in \mathfrak{B}_{a}^{ \pm}$, and therefore $N$ is contained in the kernel of $\kappa$. This proves (a). Now (b) is immediate from the definitions, and (c) follows from (a) (applied for $G=\bar{G}$ ) and (b).

Next we give an application of the existence theorem to the existence of quotients. 
5.16. Proposition. Let $(G, \psi)$ be an elementary system, and let $N$ be a normal subgroup sheaf of $G$ which is stable under $\psi$. Let $N^{ \pm}=N \cap U^{ \pm}$ and $N_{0}=N \cap H$.

(a) If $g=u v h w \in U^{+}(R) U^{-}(R) H(R) U^{+}(R)$ then $g \in N(R)$ if and only if $w u \in N^{+}(R), v \in N^{-}(R), h \in N_{0}(R)$. In particular.

$$
N \cap \Omega=N^{-} \cdot N_{0} \cdot N^{+} .
$$

(b) Let $\mathfrak{n}^{ \pm}=\operatorname{Lie}\left(N^{ \pm}\right) \subset \mathfrak{B}^{ \pm}$. Then $\left(\mathfrak{n}^{+}, \mathfrak{n}^{-}\right)$is an outer ideal (cf. [15, 1.3]) of the Jordan pair $\mathfrak{B}=\left(\mathfrak{B}^{+}, \mathfrak{B}^{-}\right)$associated with $(G, \psi)$, stable under Ad $H$.

Proof. (a) By passing to a base extension we may assume that $R=k$. Consider first the case $w=1$ and let $u=\exp (x), v=\exp (y)$. Applying $\psi_{t}$ it follows that

$$
\psi_{t}(g)=\exp (t x) \exp \left(t^{-1} y\right) h \in N(R),
$$

for all invertible $t \in R, R \in k$-alg. Since $N$ is normal, we have $\exp (-x)$ $\cdot \psi_{t}(g) \cdot g^{-1} \cdot \exp (x)=\exp ((t-1) x) \exp \left(\left(t^{-1}-1\right) y\right) \in N(R)$. If $t-1$ is invertible then by $(2), \psi_{t-1}(g) \in N(R)$, and hence

$$
\begin{aligned}
& \left(\psi_{t-1}(g)\right)^{-1} \cdot \exp ((t-1) x) \exp \left(\left(t^{-1}-1\right) y\right) \\
& \quad=h^{-1} \cdot \exp \left(\left(t^{-1}-1-(t-1)^{-1}\right) y\right) \\
& \quad=h^{-1} \cdot \exp \left(\left(\frac{t^{2}-t+1}{t(1-t)}\right) y\right) \in N(R) .
\end{aligned}
$$

Choose now $R=k[T] /\left(T^{2}-T+1\right)$ and let $t$ be the image of the indeterminate $T$ in $R$. Then $R$ is fppf over $k$, and $t^{-1}=1-t$. It follows that $h^{-1} \in N(R)$, and since $N$ is a sheaf, $h \in N(k)$. Similarly, there exists a fppf extension $R^{\prime}$ of $k$ and an element $t \in R^{\prime *}$ such that $1-t$ and $s=\left(t^{2}-t+1\right) / t(1-t)$ are invertible. Then $\exp (s y) \in N(R)$ and hence $\psi_{s-1}(\exp (s y))=\exp (y) \in N\left(R^{\prime}\right)$ which implies $v=\exp (y) \in N(k)$ as before. It follows that $u=\exp (x)=g h^{-1} \exp (y) \in N(k)$.

In the general case, $g=u v h w \in N(k)$ if and only if $w g w^{-1}=(w u) v h \in$ $N(k)$, and by what we just proved, $w u \in N^{+}(k), v \in N^{-}(k), h \in N_{0}(k)$.

(b) Since $N^{ \pm}$is a subgroup functor of $U^{ \pm}, \mathfrak{n}^{ \pm}$is a $k$-submodule of $\mathfrak{B}^{ \pm}=$Lie $\left(U^{ \pm}\right)$. From 3.6 it follows that $\left(\mathfrak{n}^{+}, \mathfrak{n}^{-}\right)$is stable under Ad $H$. Let $y \in \mathfrak{n}^{-}$and $x \in \mathfrak{B}^{+}$. Then $\exp (\varepsilon y) \in N(k(\varepsilon))$, and by 4.6 .2 we have $b(x, \varepsilon y) \exp \left(\varepsilon Q_{x} y\right) \in N(k(\varepsilon))$ which implies $Q_{x} y \in \mathfrak{n}^{+}$by (a). For $z \in \mathfrak{n}^{+}$and $x \in \mathfrak{B}^{+}, y \in \mathfrak{B}^{-}$, a similar argument using 4.6 .6 shows that $Q_{y} z \in \mathfrak{n}^{-}$and 
$B(x, y) z=z-D(x, y) z+Q_{x} Q_{y} z \in \mathfrak{n}^{+}$. Hence $D(x, y) z \in \mathfrak{n}^{+}$, and $D\left(\mathfrak{B}^{-}, \mathfrak{B}^{+}\right) \mathfrak{n}^{-}$ $\subset \mathfrak{n}^{-}$is shown similarly.

5.17. Corollary. Assume that $N$ is a smooth $k$-scheme. Then $N_{0}$, $N^{+}$, and $N^{-}$are smooth.

Proof. $N \cap \Omega$ is open in $N$ and therefore smooth. By 5.16 and 3.4, $N \cap \Omega \cong N^{-} \times N^{0} \times N^{+}$. Since $N^{ \pm}=N \cap U^{ \pm}$is a fibred product of locally finitely presented $k$-schemes it is itself locally finitely presented. Hence to show that $N^{ \pm}$is smooth, we only have to show that, for every $R \in k$ alg, and every ideal $I$ of $R$ of square zero, the canonical map $N^{ \pm}(R) \rightarrow$ $N^{ \pm}(R / I)$ is surjective (cf. [5, p. 111,4.6]). Let $n_{ \pm} \in N^{ \pm}(R / I)$. Then $n_{-} \cdot n_{+}$ $\in(N \cap \Omega)(R / I)$, and by smoothness of $N \cap \Omega$, there exists $g=u h v \in$ $N^{-}(R) N_{0}(R) N^{+}(R)$ such that $g_{R / I}=u_{R / I} h_{R / I} v_{R / I}=n_{-} \cdot n_{+}$. Hence $u_{R / I}=n_{-}$, $v_{R / I}=n_{+}$, and there assertion follows. In particular, $N^{+} \times N^{-}$is faithfully flat and locally finitely presented over $k$, and hence the projection $N \cap \Omega$ $\rightarrow N_{0}$ is faithfully flat and locally finitely presented. By EGA IV, 17.7.5 and 17.7.7, $N_{0}$ is also smooth.

5.18. Lemma. Let $\mathfrak{B}$ and $\mathfrak{B}^{\prime}$ be finite-dimensional Jordan pairs over a field, and let $f: \mathfrak{B} \rightarrow \mathfrak{B}^{\prime}$ be a surjective homomorphism. Then $f$ induces a surjection of the set of quasi-invertible pairs of $\mathfrak{B}$ onto that of $\mathfrak{B}^{\prime}$.

Proof. Let $(\bar{x}, \bar{y})$ be quasi-invertible in $\mathfrak{B}^{\prime}$ and let $(x, y) \in \mathfrak{B}^{+} \times \mathfrak{B}^{-}$be such that $f_{+}(x)=\bar{x}$ and $f_{-}(y)=\bar{y}$. Replacing $\mathfrak{B}$ and $\mathfrak{B}^{\prime}$ by the subpairs generated by $(x, y)$ and $(\bar{x}, \bar{y})$, respectively, we may assume that $\mathfrak{B}$ and $\mathfrak{B}^{\prime}$ are associative (see $[15,15.3]$ ). By $[15,15.8], \mathfrak{B} \cong \mathfrak{B}_{0} \times \mathfrak{B}_{1} \times \cdots \times \mathfrak{B}_{n}$ where $\mathfrak{B}_{0}$ is nilpotent and the $\mathfrak{B}_{1}, \cdots, \mathfrak{B}_{n}$ are local. Let $\Re$ be the kernel of $f$. Then $\Re \cong \Re_{0} \times \Re_{1} \times \cdots \times \Re_{n}$ where either $\Re_{i}=\mathfrak{B}_{i}$ or $\Re_{i} \subset \operatorname{Rad} \mathfrak{B}_{i}$, for $i=1$, $\cdots, n$, since $\mathfrak{B}_{i} / \operatorname{Rad} \mathfrak{B}_{i}$ is a division pair and hence the radical is the unique proper maximal ideal of $\mathfrak{B}_{i}$. Thus $\mathfrak{B} \cong \mathfrak{B}^{(1)} \times \mathfrak{B}^{(2)}$ and $\mathfrak{A} \cong \mathfrak{\Re}^{(1)} \times$ $\mathfrak{B}^{(2)}$ where $\mathfrak{R}^{(1)} \subset \operatorname{Rad} \mathfrak{B}^{(1)}$. Let $x^{(i)}, y^{(i)}$ be the components of $(x, y) \in \mathfrak{B}^{+} \times$ $\mathfrak{B}^{-}$in $\mathfrak{B}^{(i)}$. Then $\left.(\bar{x}, \bar{y})=f_{+}\left(x^{(1)}\right), f_{-}\left(y^{(1)}\right)\right)$ and $\left(x^{(1)}, y^{(1)}\right)$ is quasi-invertible by $[15,4.3]$.

5.19. ThEOREM. With the notations of 5.16, assume that $N$ is closed in $G$ and that $N^{+}$and $N^{-}$are smooth.

(a) $\mathfrak{n}^{ \pm}$is a direct summand of $\mathfrak{B}^{ \pm}$, the restriction exp: $\mathfrak{n}_{a}^{ \pm} \rightarrow N^{ \pm}$is an isomorphism, and $\left(\mathfrak{n}^{+}, \mathfrak{n}^{-}\right)$is an ideal of $\mathfrak{B}$, stable under $\operatorname{Ad} H$.

(b) Let $G^{\prime}=G / N$ (quotient sheaf), and let $\psi^{\prime}$ be the action of $k_{m}$ on 
$G^{\prime}$ induced by $\psi$. Then $\left(G^{\prime}, \psi^{\prime}\right)$ is an elementary system, the canonical map $f:(G, \psi) \rightarrow\left(G^{\prime}, \psi^{\prime}\right)$ is a homomorphism, and the Jordan pair associated with $\left(G^{\prime}, \psi^{\prime}\right)$ is $\mathfrak{B}^{\prime}=\left(\mathfrak{B}^{+} / \mathfrak{n}^{+}, \mathfrak{B}^{-} / \mathfrak{n}^{-}\right)$.

Proof. (a) Since $N$ is closed in $G, N^{+}=N \cap U^{+}$is closed in $U^{+}$ and hence is an affine scheme. Let $\omega_{N+/ k}$ be the pullback of the module of differentials $\Omega_{N^{+/ k}}^{1}$ to $k$ via the unit section. Smoothness of $N^{+}$implies that $\omega_{N+/ k}$ is a finitely generated and projective $k$-module, and so is $\mathfrak{t}^{+}=$ Lie $\left(N^{+}\right)=\operatorname{Hom}_{k}\left(\omega_{N+/ k}, k\right.$ ) (cf. [7. p. 208, and p. 215]). From SGA3, Exp. II, 4.11, it follows that $\mathfrak{n}^{+}$is a direct summand of $\mathfrak{B}^{+}$. Let $\exp (x) \in N^{+}(R)$. Then $\psi_{1+\varepsilon}(\exp (x))=\exp ((1+\varepsilon) x)=\exp (x) \exp (\varepsilon x) \in N^{+}(R(\varepsilon))$ and hence $\exp (\varepsilon x) \in N^{+}(R(\varepsilon))$; i,e., $x \in \operatorname{Lie}\left(N_{R}^{+}\right)=\mathfrak{n}_{R}^{+}$. This shows that $N^{+}$is contained in the vector group $U=\exp \left(\mathfrak{n}_{a}^{+}\right) \cong \mathfrak{n}_{a}^{+}$. Since $U$ and $N^{+}$are smooth and have the same Lie algebra (namely $\mathfrak{n}^{+}$) the fibres of $U$ and $N^{+}$have the same dimension. By EGA IV, 17.11.5, $N^{+}$is open in $U$. Arguing fibrewise, it follows easily that $N^{+}=U$. The proof for $N^{-}$is the same. Let $x \in \mathfrak{n}^{+}$and $y \in \mathfrak{B}^{-}$. Then $\exp (x) \in N^{+}(k)$, and by 4.6.2, $Q_{x} y \in \mathfrak{n}^{+}$. Similarly, one shows that $Q\left(\mathfrak{n}^{-}\right) \mathfrak{B}^{+} \subset \mathfrak{n}^{-}$, and together with 5.16 (b) it follows that $\left(\mathfrak{n}^{+}, \mathfrak{n}^{-}\right)$is an ideal of $\mathfrak{B}$.

(b) We will construct a Jordan system $\mathscr{J}^{\prime}=\left(\mathfrak{B}^{\prime}, H^{\prime}, \rho^{\prime}, b^{\prime}\right)$ whose associated elementary system is $\left(G^{\prime}, \psi^{\prime}\right)$. Let $\mathfrak{B}^{\prime}=\left(\mathfrak{B}^{+} / \mathfrak{n}^{+}, \mathfrak{B}^{-} / \mathfrak{n}^{-}\right)$. Then $\mathfrak{B}^{\prime}$ is a Jordan pair over $k$ which is finitely generated and projective as a $k$ module since $\mathfrak{n}^{ \pm}$is a direct summand of $\mathfrak{B}^{ \pm}$. Let $H^{\prime}=H / N_{0}$ (quotient sheaf). Since $N_{0}=N \cap H$ is closed in $H$, the equivalence relation on $H$ defined by $N_{0}$ is closed in $H \times H$, and by $1.3, H^{\prime}$ is a separated $k$-group sheaf. It is easily checked that there is a unique homomorphism $\rho^{\prime}: H^{\prime}$ $\rightarrow$ Aut $\left(\mathfrak{B}^{\prime}\right)$ satisfying

$$
\rho_{ \pm}^{\prime}\left(h^{\prime}\right) \cdot x^{\prime}=(\operatorname{Ad} h \cdot x)^{\prime}
$$

where the ' denotes the canonical maps $H \rightarrow H^{\prime}$ and $\mathfrak{B} \rightarrow \mathfrak{B}^{\prime}$. The canonical map $\mathfrak{B} \rightarrow \mathfrak{B}^{\prime}$ is surjective and linear. Hence the induced morphism on the quasi-invertible pairs $W \rightarrow W^{\prime}$ is smooth, and by 5.18 , it is surjective. In particular, it is faithfully flat and locally finitely presented and therefore an epimorphism of sheaves ([5, p. 295, 2.10]). This allows us to define a morphism $b^{\prime}: W^{\prime} \rightarrow H^{\prime}$ by

$$
b^{\prime}\left(x^{\prime}, y^{\prime}\right)=b(x, y)^{\prime},
$$

provided we can show that the right hand side depends only on the 
equivalence class of $(x, y) \bmod \left(\mathfrak{n}^{+}, \mathfrak{n}^{-}\right)$. Thus let $(x, y) \in W(R)$, and assume that $x \equiv u\left(\mathfrak{n}_{R}^{+}\right)$and $y^{x} \equiv v\left(\mathfrak{n}_{R}^{-}\right)$. Since $\left(\mathfrak{n}_{R}^{+}, \mathfrak{n}_{R}^{-}\right)$is an ideal of $\mathfrak{B}_{R}$ we have $x^{y} \equiv u^{v}\left(\mathfrak{n}_{R}^{+}\right)$and $y^{x} \equiv v^{u}\left(\mathfrak{n}_{R}^{-}\right)$, and since $\exp \mathfrak{n}_{a}^{ \pm}=N^{ \pm}$, it follows from $(*)$ of 4.1 that

$$
\begin{aligned}
b(x, y) & =\exp \left(-y^{x}\right) \exp (x) \exp (y) \exp \left(-x^{y}\right) \\
& \equiv \exp \left(-v^{u}\right) \exp (u) \exp (v) \exp \left(-u^{v}\right) \\
& =b(u, v) \text { modulo } N(R) .
\end{aligned}
$$

Now it is easily verified that $\mathscr{J}^{\prime}=\left(\mathfrak{B}^{\prime}, H^{\prime}, \rho^{\prime}, b^{\prime}\right)$ is a Jordan system, and that the canonical maps $\mathfrak{B} \rightarrow \mathfrak{B}^{\prime}$ and $H \rightarrow H^{\prime}$ define a homomorphism $\Phi: \mathscr{J} \rightarrow \mathscr{J}^{\prime}$ where $\mathscr{J}$ is the Jordan system associated with $(G, \psi)$. Let $\left(G^{\prime}, \psi^{\prime}\right)$ be the elementary system defined by $\mathscr{J}^{\prime}$, and let $f:(G, \psi) \rightarrow\left(G^{\prime}, \psi^{\prime}\right)$ be the homomorphism induced by $\Phi$ (see 4.15 and 5.1). Then $f: G \rightarrow G^{\prime}$ is an epimorphism of group sheaves, and we only have to check that $\operatorname{Ker}(f)$ $=N$. By 5.16 (a), it suffices to show that $\Omega \cap \operatorname{Ker}(f)=\Omega \cap N$, and this is clear from the definitions.

5.20. EXAMPLE. If $N^{+}$and $N^{-}$are not smooth then the induced action $\psi^{\prime}$ on $G / N$ may not be elementary. For example, let $k$ be a field, let $G$ $=\mathbf{S O}_{2 n+1}$ be the special orthogonal group of the quadratic form $q\left(x_{0}, \cdots\right.$, $\left.x_{2 n}\right)=x_{0}^{2}+\sum x_{i} x_{n+i}$, and let $\psi_{t}$ be conjugation by the matrix

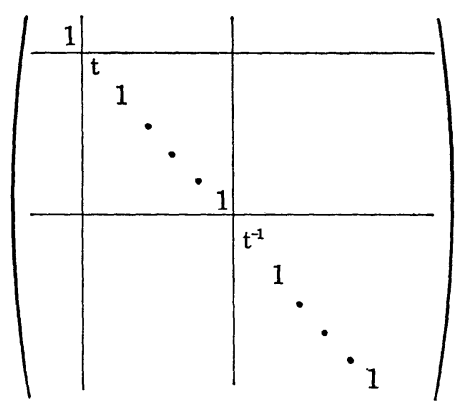

Then $\psi$ is an elementary action whose associated Jordan pair is the simple Jordan pair defined by the standard quadratic form on $k^{2 n-1}$ (cf. [18, p. 196]). Now let $\operatorname{char}(k)=2$, and let $f: G \rightarrow \mathbf{S} \mathbf{p}_{n, k}$ be the exceptional isogeny

$$
\left(\begin{array}{lll}
1 & a & b \\
0 & A & B \\
0 & C & D
\end{array}\right) \longmapsto\left(\begin{array}{ll}
A & B \\
C & D
\end{array}\right)
$$


where $a, b \in k^{n}$ and $A, B, C, D$ are $n \times n$ matrices. Then $N=\operatorname{Ker}(f)$ is an infinitesimal group of height one, and $\left(\mathfrak{n}^{+}, \mathfrak{n}^{-}\right)$is the unique proper onedimensional outer ideal of $\mathfrak{B}$. The induced action $\psi^{\prime}$ on $G^{\prime}=\mathbf{S p}_{n, k}$ is not elementary since it has weights $0, \pm 1, \pm 2$ on Lie $\left(G^{\prime}\right)$.

\section{§6. The radical}

6.1. In this section, $k$ denotes a field and $\bar{k}$ an algebraic closure of $k$. A $k$-group is an affine finitely presented group scheme over $k$. If $(G, \psi)$ is an elementary system we will always assume that $G$ is a $k$-group. By 5.13 , so is $H$, and conversely, if $(\mathfrak{B}, H, \rho, b)$ is a Jordan system with $H$ a $k$-group then the associated group $G$ is a $k$-group. In particular, the projective group of $\mathfrak{B}$ is a $k$-group.

Radical and unipotent radical of a smooth connected $\bar{k}$-group are denoted by $R(G)$ and $R_{u}(G)$. If $G$ is not connected define $R(G)=R\left(G^{0}\right)$ and $R_{u}(G)=R_{u}\left(G^{0}\right)$. By definition, a $k$-group is semisimple (reductive) if it is smooth and $R\left(G_{\bar{k}}\right)=\{1\}\left(R_{u}\left(G_{\bar{k}}\right)=\{1\}\right)$. Let $S$ be a torus acting on a $k$-group $G$ by automorphisms. If $G$ is connected (smooth, reductive) so is the fixed point set $G^{S}$; more generally, $R_{u}\left(G^{S}\right)=R_{u}(G)^{S}$ (SGA3, Exp. XIX).

6.2. Let $\mathfrak{B}$ be a Jordan pair over $k$. An element $x \in \mathfrak{B}^{+}$is called properly quasi-invertible if $(x, y)$ is quasi-invertible for all $y \in \mathfrak{B}^{-}$. Similarly one defines properly quasi-invertible for elements of $\mathfrak{B}^{-}$. The Jacobson radical of $\mathfrak{B}$ is $\operatorname{Rad} \mathfrak{B}=\left(\operatorname{Rad} \mathfrak{B}^{+}, \operatorname{Rad} \mathfrak{B}^{-}\right)$where $\operatorname{Rad} \mathfrak{B}^{\sigma}$ is the set of properly quasi-invertible elements of $\mathfrak{B}^{\circ}$ (cf. $\left.[15, \S 4]\right)$. We say $\mathfrak{B}$ is semisimple if $\operatorname{Rad} \mathfrak{B}=0$ and $\mathfrak{B}$ is separable if $\mathfrak{B}_{K}$ is semisimple, for all extension fields $K$ of $k$. An ideal $\mathfrak{S}=\left(\mathfrak{S}^{+}, \mathfrak{S}^{-}\right)$of $\mathfrak{B}$ is called trivial if $Q\left(\mathfrak{S}^{ \pm}\right) \cdot \mathfrak{S}^{\mp}=0$. Clearly a trivial ideal is contained in $\operatorname{Rad} \mathfrak{B}$.

6.3. Lemma. Let $(G, \psi)$ be an elementary system over $k$, and let $N$ be a smooth connected abelian normal subgroup of $G$, invariant under $\psi$. Then (with the notation of 5.16) $N=N^{-} \cdot N_{0} \cdot N^{+} \subset \Omega$, and $\left(\mathfrak{n}^{+}, \mathfrak{n}^{-}\right)$is a trivial ideal of the associated Jordan pair $\mathfrak{B}$.

Proof. Since $N$ is commutative, $N \cap \Omega=N^{-} \cdot N_{0} \cdot N^{+}$is an open subgroup of $N$. Hence $N=N \cap \Omega$. By 5.17 and 5.19, $N^{ \pm}=\exp \left(\mathfrak{n}_{a}^{ \pm}\right)$and $\left(\mathfrak{n}^{+}, \mathfrak{n}^{-}\right)$ is an ideal of $\mathfrak{B}$. If $x \in \mathfrak{n}^{+}, y \in \mathfrak{n}^{-}$then $\exp (x) \exp (y)=\exp (y) \exp (x)=$ $\exp \left(y^{x}\right) b(x, y) \exp \left(x^{y}\right)$ implies $y=y^{x}, b(x, y)=1, x=x^{y}$. Hence $x=x^{y}=$ $B(x, y)^{-1} \cdot\left(x-Q_{x} y\right)=x-Q_{x} y$ and thus $Q_{x} y=0$. Similarly $Q_{y} x=0$. 
6.4. THEOREM. The projective group $G=\mathbf{P G}(\mathfrak{B})$ of a separable Jordan pair $\mathfrak{B}$ is semisimple and its identity component is generated by $U^{+}$and $U^{-}$ (i.e., $G_{i n}=G^{0}$; cf. 3.9).

Proof. We may assume $k$ algebraically closed. Let $S$ be as in 5.15, and $G^{\prime}=S \cdot G_{i n}$. Then $G^{\prime}$ is a smooth connected $k$-group which is normal in $G$. We claim that $G^{\prime}$ is semisimple. If this were not so, let $N$ be the last non-trivial term in the derived series of $R\left(G^{\prime}\right)$. By 6.3. $\left(\mathfrak{n}^{+}, \mathfrak{n}^{-}\right)$is then a trivial ideal of $\mathfrak{B}$ and hence contained in $\operatorname{Rad}(\mathfrak{B})=0$. Hence $N^{ \pm}=\{1\}$ and $N=N_{0} \subset H$. But $H$ contains no nontrivial normal subgroups of $G$ by 5.15 . Hence $N=\{1\}$, a contradiction. Now $G_{i n}$ is normal in $G^{\prime}$ and hence is semisimple as well. Since $G^{\prime} / G_{i n}$ is at most one-dimensional we have $G_{i n}=G^{\prime}$; i.e., $S \subset G_{i n}$. Now consider the homomorphism $f: G \rightarrow$ Aut $\left(G_{i n}\right)=A$ given by $g \mapsto \operatorname{Int}(g)$. Then $f$ is a monomorphism. Indeed, since $S \subset G_{i n}$, the kernel of $f$ is contained in $G^{S}=H$ and is therefore trivial (5.15). By SGA3, Exp. XXIV, $A$ is a semisimple $k$-group, and $A^{0}=$ $f\left(G_{i n}\right) \subset f(G)$. Hence $f(G) \cong G$ is an open subgroup of $A$, in particular, it is semisimple. Also, $G^{0} \cong f\left(G^{0}\right)=A^{0}=f\left(G_{i n}\right)$ and therefore $G^{0}=G_{i n}$.

6.5. Corollary. Let $\mathfrak{B}$ be a separable Jordan pair over $k$. Then Aut() is a reductive $k$-group whose identity component is Inn()).

Proof. Since Aut $(\mathfrak{B})=H=G^{S}$ and $\operatorname{Inn}(\mathfrak{B})=H_{i n}$ where $G$ is the projective group of $\mathfrak{B}$, this follows from 6.4 and 4.12. From 2.6 we get

6.6. CoRollary. Let $\mathfrak{A}$ be a finite-dimensional separable unital quadratic Jordan algebra over $k$. Then the structure group $\operatorname{Str}(\mathfrak{H})$ is $a$

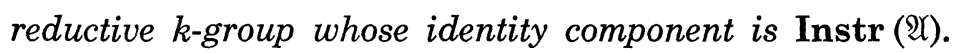

This was proved by Springer in [20] by a case-by-case verification.

6.7. Proposition. Let $(G, \psi)$ be an elementary system over $k$, and assume that the associated Jordan pair $\mathfrak{B}$ is separable. Then the automorphisms $\psi_{t}$ are inner in the following sense: There exists a $k$-split torus $S \subset H_{\text {in }}$ and a nontrivial character $\eta: S \rightarrow k_{m}$ such that

$$
\operatorname{Int}(s)=\psi_{\eta(s)},
$$

for all $s \in S$.

Proof. Let $\kappa: G \rightarrow \bar{G}=\mathbf{P G}(\mathfrak{B})$ be the canonical homomorphism, and let $\bar{S}=\gamma\left(k_{m}\right) \subset \bar{G}^{0}$ as in 5.15. By 6.4, $\bar{G}^{0}=\bar{G}_{i n}$, and hence the restriction 
$\kappa_{0}: G_{i n} \rightarrow \bar{G}^{0}$ is an epimorphism. Let $Z=\kappa_{0}^{-1}(\bar{S}) \subset H_{i n}$, and define $\eta: Z \rightarrow$ $k_{m}$ by $\kappa_{0}(s)=\gamma(\eta(s))$. For $x \in \mathfrak{B}_{a}^{ \pm}$we then have

$$
\operatorname{Int}(s) \cdot \exp (x)=\exp (\operatorname{Ad} s \cdot x)=\exp \left(\eta(s)^{ \pm 1} \cdot x\right),
$$

since $\kappa$ induces isomorphisms $U^{ \pm} \rightarrow \bar{U}^{ \pm}$(cf. 5.15). It follows that $s b(x, y) s^{-1}$ $=b(\operatorname{Ad} s \cdot x, \operatorname{Ad} s \cdot y)=b(x, y)$, and hence $Z$ is central in $H_{i n}$. Now $\kappa\left(H^{0}\right)$ $=\kappa\left(H_{i n}\right)=\bar{H}^{0}$ implies that $H^{0} \subset \operatorname{Ker}(\kappa) \cdot H_{i n}$. But $\operatorname{Ker}(\kappa)$ centralizes $G_{i n}$, and hence $Z$ is central in $H^{0}$. Let $S$ be the maximal $k$-split torus of the centralizer of $H$ in $Z$. Then $S$ is central in $H$, and $\kappa: S \rightarrow \bar{S}$ is an epimorphism. Hence $\eta: S \rightarrow k_{m}$ is nontrivial. Now (1) follows from (2) and the fact that $S$ is central in $H$.

6.8. Lemma. Let $(G, \psi)$ be an elementary system over $k$ and assume that $G$ is reductive and connected. Then $P^{+}=H \cdot U^{+}$and $P^{-}=H \cdot U^{-}$are opposed parabolic subgroups of $G$.

Proof. Let $\hat{G}=G \times k_{m}$ (semidirect product) and let $S=\{1\} \times k_{m} \subset \hat{G}$. Then $\hat{G}$ is reductive, and $S$ is a one-dimensional split torus of $\hat{G}$ with root system $\Phi=\mathrm{A}_{1}$. Hence $\hat{G}_{\Phi^{+}}=S \cdot P^{+}$and $G_{\Phi^{-}}=S \cdot P^{-}$are opposed parabolic subgroups of $\hat{G}([3,4.15])$, and hence $P^{+}=G \cap\left(S \cdot P^{+}\right)$and $P^{-}=G \cap\left(S \cdot P^{-}\right)$ are opposed parabolic subgroups of $G$.

6.9. Theorem. Let $(G, \psi)$ be an elementary system over an algebraically closed field $k$, and assume that $G$ is smooth and connected. Let $\operatorname{Rad}(\mathfrak{B})$ $=\left(\mathfrak{n}^{+}, \mathfrak{n}^{-}\right)$be the radical of the associated Jordan pair $\mathfrak{B}$, and let $N^{ \pm}=$ $\exp \left(\mathfrak{n}_{a}^{ \pm}\right) \subset U^{ \pm}$. Then $R_{u}(G)=N^{-} \cdot R_{u}(H) \cdot N^{+}$.

Proof. Let $N_{0}$ be the $k$-subgroup of $H$ generated by $b\left(\mathfrak{B}_{a}^{+}, \mathfrak{n}_{a}^{-}\right)$and $b\left(\mathfrak{n}_{a}^{+}, \mathfrak{B}_{a}^{-}\right)$. This is a smooth connected $k$-group. We claim that

$$
N=N^{-} \cdot N_{0} \cdot N^{+}
$$

is a smooth connected unipotent normal subgroup of $G$; in particular, $N \subset R_{u}(G)$. Clearly, $N$ is smooth and connected. To show that it is a normal subgroup of $G$ it suffices to show that $N(k)$ is normal in $G(k)$, since $k$ is algebraically closed, and all groups involved are smooth. From 4.1 and the definition of $\operatorname{Rad}(\mathfrak{B})$ it follows that $N(k)$ is a subgroup of $G(k)$. The invariance of $\operatorname{Rad}(\mathfrak{B})$ under Aut $(\mathfrak{B})$ implies that $N(k)$ is stable under conjugation by $H(k)$. If $x \in \mathfrak{B}^{+}$and $y \in \mathfrak{n}^{-}$then

$$
\operatorname{Int}(\exp (x)) \cdot \exp (y)=\exp \left(y^{x}\right) \cdot b(x, y) \cdot \exp \left(x^{y}-x\right)
$$


with $y^{x}=y+Q_{y} \cdot x^{y} \in \mathfrak{n}^{-}$and $x^{y}-x=Q_{x} \cdot y^{x} \in \mathfrak{n}^{+}$, since $\operatorname{Rad}(\mathfrak{B})$ is an ideal of $\mathfrak{B}$. Also $b(x, y) \in N_{0}(k)$. If $u \in \mathfrak{B}^{+}, v \in \mathfrak{n}^{-}$, or $u \in \mathfrak{n}^{+}, v \in \mathfrak{B}$ then by 3.6 and 4.1 ,

$$
\operatorname{Int}(\exp (x)) \cdot b(u, v)=\exp (x-B(u, v) \cdot x) \cdot b(u, v),
$$

and $x-B(u, x) \cdot x=-\{u v x\}+Q_{u} Q_{v} x \in \mathfrak{n}^{+}$, again since $\operatorname{Rad}(\mathfrak{B})$ is an ideal. Thus $N(k)$ is stable under conjugation by $U^{+}(k)$, and similarly one shows that it is stable under conjugation by $U^{-}(k)$. This proves that $N$ is normal in $G$. Now we show that $N$ is unipotent. Replacing $G$ by $G / R_{u}(G)$ (which is permissible by 5.19), we may assume that $G$ is reductive, and then have to show that $N=\{1\}$. By $6.8, P^{+}$and $P^{-}$are parabolic subgroups of $G$, and hence $N \cap P^{+}=N_{0} \cdot N^{+}$and $N \cap P^{-}=N_{0} \cdot N^{-}$are parabolic subgroups of $N$. Then $N / N_{0} \cdot N^{ \pm} \cong N^{\mp}$ is both affine and projective which implies $N^{ \pm}=\{1\}$ and therefore also $N_{0}=\{1\}$.

Let now $N^{\prime}=N^{-} \cdot R_{u}(H) \cdot N^{+}$. By $6.2, \quad N_{0}=N \cap H \subset R_{u}(G) \cap H=$ $R_{u}(H)$ and hence $N \subset N^{\prime}$. Since $N^{+} \cdot N^{-} \subset N \subset N^{\prime}$ it follows that $N^{\prime}$ is a subgroup of $G$. Also, $N^{\prime}$ is unipotent since $N$ and $N^{\prime} / N=R_{u}(H) / N_{0}$ are unipotent. We claim that $N^{\prime}$ is normal in $G$. In view of what we proved before, it suffices to show that conjugation by elements of $U^{ \pm}(k)$ maps $R_{u}(H)$ into $N^{\prime}$. Let $x \in \mathfrak{B}^{ \pm}$and $h \in R_{u}(H)(k)$. Then

$$
\operatorname{Int}(\exp (x)) \cdot h=\exp (x-\operatorname{Ad} h \cdot x) \cdot h \text {. }
$$

and we have to show that $x-\operatorname{Ad} h \cdot x \in \mathfrak{n}^{ \pm}$. Consider the homomorphism $\varphi: G \rightarrow G^{\prime}=\mathbf{P G}(\mathfrak{B} / \operatorname{Rad}(\mathfrak{B}))^{0}$ induced by the canonical homomorphisms $\mathfrak{B}$ $\rightarrow \mathfrak{B} / \operatorname{Rad}(\mathfrak{B})$ and $H \rightarrow \operatorname{Aut}(\mathfrak{B}) \rightarrow \operatorname{Aut}(\mathfrak{B} / \operatorname{Rad}(\mathfrak{B}))$ (cf. 4.15). By 6.4, $G^{\prime}$ is semisimple, and $G^{\prime}=G_{i n}^{\prime}$. Hence $\varphi$ is an epimorphism, and we have $R_{u}(H) \subset R_{u}(G) \subset \operatorname{Ker}(\varphi)$. Applying $\varphi$ to (1) it follows that $\operatorname{Lie}(\varphi)$ $(x-\operatorname{Ad} h \cdot x)=0$, and hence $x-\operatorname{Ad} h \cdot x \in \mathfrak{n}^{ \pm}$. This proves that $N^{\prime}$ is normal in $G$ and therefore contained in $R_{u}(G)$. To prove equality, we show that $G / N^{\prime}$ is reductive. Replacing $G$ by $G / N^{\prime}$ (which we can do in view of 5.19), we may assume that $H$ is reductive and $\mathfrak{V}$ is separable, and then have to show that $G$ is reductive. Consider the canonical homomorphism $\kappa: G \rightarrow \mathbf{P G}(\mathfrak{B})^{0}$. Then by 6.4 and $5.15, R_{u}(G) \subset \operatorname{Ker}(\kappa) \subset H$ which shows $R_{u}(G)=\{1\}$. This completes the proof.

6.10. Corollary. (a) $\mathfrak{B}$ is separable if and only if the projective group $G$ of $\mathfrak{B}$ is semisimple.

(b) $\mathfrak{B}=\operatorname{Rad} \mathfrak{B}$ if and only if the "inner projective group" $G_{i n}$ is uni- 
potent.

6.11. COROLLARY. Let $\mathfrak{B}$ be a Jordan pair over an algebraically closed field with non-zero radical. Then $\mathfrak{B}$ contains a nonzero trivial ideal, invariant under all automorphisms and antiautomorphisms of $\mathfrak{B}$.

Proof. Let $G=\mathbf{P G}(\mathfrak{B})$. By $6.9, G_{i n}$ is not semisimple. Let $N$ be the last non-trivial term in the derived series of $R\left(G_{i n}\right)$, and let $N=N^{-} \cdot N_{0} \cdot N^{+}$ as in 6.3. Then $N^{+}$and $N^{-}$are not both equal to $\{1\}$. Otherwise, $N=N_{0}$ would be a normal subgroup of $G$ contained in $H$ and therefore $N_{0}=\{1\}$. Then $\left(\mathfrak{n}^{+}, \mathfrak{n}^{-}\right)$has the desired properties.

Remark. This results was used in [14] to prove the nilpotence of the radical of a Jordan triple system. A direct proof can now be found in [15].

6.12. Theorem. Let $(G, \psi)$ be an elementary system over $k$ and let $K$ be an extension field of $k$. Then

$$
G(K)=U^{+}(k) \cdot \Omega(K)=\Omega(K) \cdot U^{-}(k) .
$$

Proof. (a) $k$ infinite. Let $\bar{K}$ be the algebraic closure of $K$, and let $\Omega^{*}=U^{-}(k) \cdot H(K) \cdot U^{+}(K)$. Then $\Omega^{*}$ is Zariski-dense in $G(\bar{K})$. Indeed, let $\pi: X \rightarrow G$ be as in 4.13. Then $\pi: X(\bar{K}) \rightarrow G(\bar{K})$ is surjective ([5, p. 291, 1.15]), and $\pi^{-1}\left(\Omega^{*}\right)$ contains $W(k) \times H(\bar{K}) \times \mathfrak{B}_{\bar{K}}^{ \pm}$(where $W \subset \mathfrak{B}_{a}^{+} \times \mathfrak{B}_{a}^{-}$is the subscheme of quasi-invertible pairs). Since $k$ is infinite, $W(k)$ is Zariski-dense in $\mathfrak{B}_{\bar{K}}^{+} \times \mathfrak{B}_{\bar{K}}^{-}$and hence $\pi^{-1}\left(\Omega^{*}\right)$ is Zariski-dense in $X(\bar{K})$. It follows that $\Omega^{*}$ is Zariski-dense in $G(\bar{K})$. Now let $g \in G(K)$. Then $\left(g \cdot \Omega^{*}\right) \cap \Omega(\bar{K})$ is not empty since $\Omega(\bar{K})$ is Zariski-open in $G(\bar{K})$. Hence there exist $u \in U^{-}(k)$, $h \in H(\bar{K}), \quad v \in U^{+}(\bar{K}), \omega \in \Omega(\bar{K})$ such that $g u h v=\omega$. This implies $g u=$ $\omega v^{-1} h^{-1} \in \Omega(\bar{K}) \cap G(K)=\Omega(K)$, and hence $g=\left(\omega v^{-1} h^{1}\right) u^{-1} \in \Omega(K) \cdot U^{-}(k)$. By passing to $\left(G, \psi^{-1}\right)$, we get the other equation.

(b) $k$ finite. Let us first assume that $G$ is reductive and connected. Then by $6.8, P^{+}$and $P^{-}$are opposed parabolic subgroups of $G$, and by 6.9 , $\mathfrak{B}$ is separable. Choose a split torus $S$ as in 6.7, and let $S^{\prime}$ be a maximal $k$-split torus of $G$ containing $S$. Let $P^{\prime+}$ and $P^{\prime-}$ be the opposed minimal parabolic subgroups of $G$ defined by $S^{\prime}$. Then $P^{\prime \sigma} \subset P^{\prime \sigma}$, and by [3, 6.25], $G(K)=P^{\prime+}(k) \cdot P^{\prime-}(K) \cdot P^{\prime+}(K)=P^{+}(k) \cdot P^{-}(K) \cdot P^{+}(K)=U^{+}(k) \cdot \Omega(K)$.

Now let $G$ be arbitrary. We have $G=H \cdot U^{+} \cdot U^{-} \cdot U^{+}=H \cdot G_{i n}=G_{i n} \cdot H$, and the kernel of the homomorphism $G_{i n} \rtimes H \rightarrow G$ given by multiplication 
is isomorphic with $H \cap G_{i n}=H_{i n}$ (4.12), in particular, it is connected. Hence if $K$ is finite, $G(K)=G_{i n}(K) \cdot H(K)$ by [5, p. 426, 7.6], and if $K$ is infinite, this is still true by (a). Replacing $G$ by $G_{i n}$, we may therefore assume that $G$ is smooth and connected. Let $G^{\prime}=G / R_{u}(G)$ and let $\psi^{\prime}$ be the action induced by $\psi$ on $G^{\prime}$. Then $G^{\prime}$ is reductive, $\left(G^{\prime}, \psi^{\prime}\right)$ is an elementary system, and the canonical map $\varphi:(G, \psi) \rightarrow\left(G^{\prime}, \psi^{\prime}\right)$ is a homomorphism (5.19). By 6.9, $R_{u}(G)=N^{-} \cdot R_{u}(H) \cdot N^{+}$, and $U^{\prime \sigma}=U^{\sigma} / N$ and $H^{\prime}$ $=H / R_{u}(H)$. Since $U^{\sigma} \rightarrow U^{\prime}$ is induced by the surjective linear maps $\mathfrak{B}^{\sigma}$ $\rightarrow \mathfrak{B}^{\sigma} / \operatorname{Rad}\left(\mathfrak{B}^{\sigma}\right)$, the maps $U^{\sigma}(k) \rightarrow U^{\prime \sigma}(k)$ and $U^{\sigma}(K) \rightarrow U^{\prime \sigma}(K)$ are surjective. Let now $g \in G(K)$. Then $\varphi(g) \in G^{\prime}(K)=U^{\prime+}(k) \cdot \Omega^{\prime}(K)$, by what we proved before. Hence there exist $u \in U^{+}(k), v \in U^{-}(K), w \in U(K)$ such that $\varphi(g)$ $\equiv \varphi(u v w)$ modulo $H^{\prime}(K)$, in other words, $w^{-1} v^{-1} u^{-1} g \in \varphi^{-1}\left(H^{\prime}\right)(K)$. We have $\varphi^{-1}\left(H^{\prime}\right)=N^{-} \cdot H \cdot N^{+}$. Indeed, since: $\varphi: H \rightarrow H^{\prime}$ is an epimorphism, $\varphi^{-1}\left(H^{\prime}\right)$ $H \cdot R_{u}(G)=N^{-} \cdot H \cdot N^{+}$. It follows that $w^{-1} v^{-1} u^{-1} g=x y h$ where $x \in N^{-}(K)$, $h \in H(K), y \in N^{+}(K)$, or $g=u v w x h y$. Since $N^{-}(K)=\exp \left(\operatorname{Rad}\left(\mathfrak{B}_{K}^{-}\right)\right)$it follows from 4.1 and 6.2 that $w x \in \Omega(K)$. Hence $g \in U^{+}(k) \cdot U^{-}(K) \cdot \Omega(K)$. $H(K) \cdot N^{+}(K)=U^{+}(k) \cdot \Omega(K)$. The second formula follows by passing to $\left(G, \psi^{-1}\right)$.

6.13. Corollary. (Generators and relations for $G(k)$ ). Let $k$ be infinite. Let $\Gamma$ be an abstract group, and let $\varphi_{0}: H(k) \rightarrow \Gamma, \varphi_{ \pm}: U^{ \pm}(k) \rightarrow \Gamma$ be homomorphisms. Then there exists a homomorphism $\varphi: G(k) \rightarrow \Gamma$ extending $\varphi_{0}$ and $\varphi_{+}, \varphi_{-}$if and only if

$$
\begin{gathered}
\varphi_{0}(h) \varphi_{\sigma}(u) \varphi_{0}(h)^{-1}=\varphi_{\sigma}\left(h u h^{-1}\right), \\
\varphi_{+}(\exp (x)) \varphi_{-}(\exp (y))=\varphi_{-}\left(\exp \left(y^{x}\right)\right) \varphi_{0}(b(x, y)) \varphi_{+}\left(\exp \left(x^{y}\right)\right),
\end{gathered}
$$

for all $h \in H(k), u \in U^{o}(k),(x, y) \in \mathfrak{B}^{+} \times \mathfrak{B}^{-}$quasi-invertible. Indeed, $G(k)$ $=U^{+}(k) \cdot \Omega(k)$ by 6.12 , and hence $G(k)$ is the quotient of $\mathfrak{B}^{+} \times \mathfrak{B}^{-} \times H(k)$ $\times \mathfrak{B}^{+}$by the equivalence relation given in 4.13. We can now copy the proof of 4.14, provided we show: for all $u, w \in U^{-}(k), v \in U^{+}(k)$ there exists $x \in U^{+}(k)$ such that $x u^{-1}$ and $x v w$ are in $\Omega(k)$. In view of 4.1, this amounts to showing that for all $a, c \in \mathfrak{B}^{-}, b \in \mathfrak{B}^{+}$there exists $z \in \mathfrak{B}^{+}$such that $(z,-a)$ and $(z+b, c)$ are quasi-invertible. Since $k$ is infinite, this is always possible.

6.14. As an application, we determine the relation with the group $\Xi$ introduced by Koecher in [11]. Similar remarks apply to the groups studied in $[12,13]$. Let $\mathfrak{U}$ be a finite-dimensional quadratic Jordan algebra 
with unit element $e$ over an arbitrary field $k$, and let $\Xi(\mathfrak{R})$ be the group of birational transformations of $\mathfrak{A}$ generated by the structure group $\operatorname{Str}(\mathfrak{C})$ and the maps $x \mapsto t_{y}(x)=x+y \quad(y \in \mathfrak{U})$ and $x \mapsto j(x)=-x^{-1}$. Let $\mathfrak{B}=$ $(\mathfrak{A}, \mathfrak{U})$ be the Jordan pair defined by $\mathfrak{X}$ and $G=$ PG(⿻上) the projective group of $\mathfrak{B}$. Thus $H=\operatorname{Aut}(\mathfrak{B})$. Then $\Xi(\mathfrak{U})$ is isomorphic with $G(k)$. Indeed, let $K$ be an infinite extension field of $k$, let $\varphi_{0}: H(K)=\operatorname{Aut}\left(\mathfrak{B}_{K}\right) \rightarrow$ $\operatorname{Str}\left(\mathfrak{H}_{K}\right)$ be the isomorphism $\left(g, g^{\sharp-1}\right) \mapsto g$ (cf. 2.6), and define. $\varphi_{ \pm}: U^{ \pm}(K) \rightarrow$ $\Xi\left(\mathfrak{U}_{K}\right)$ by $\varphi_{+}(\exp x)=t_{x}$ and $\varphi_{-}(\exp y)=j \circ t_{y} \circ j=\tilde{t}_{y}$. For $g \in \operatorname{Str}\left(\mathfrak{A}_{K}\right)$ we have $g \circ t_{x} \circ g^{-1}=t_{g x}$ and $j \circ g \circ j=g^{\sharp-1}$. This implies (1) of 6.13. For (2), we have to show that $t_{x} \circ \tilde{t}_{y}=\tilde{t}_{y x} \circ B(x, y) \circ t_{x y}$. Taking inverses, this is equivalent with $\tilde{t}_{y} \circ t_{x}=t_{x y} \circ B(x, y)^{-1} \circ \tilde{t}_{y^{x}}$. Now $\tilde{t}_{y}(z)=\left(z^{-1}-y\right)^{-1}=z^{y}([15$, 3.13]), and hence

$$
\left(\tilde{t}_{y} \circ t_{x}\right)(z)=(x+z)^{y}=x^{y}+B(x, y)^{-1} \cdot z^{\left(y^{x}\right)}=\left(t_{x^{y}} \circ B(x, y)^{-1} \circ \tilde{t}_{y^{x}}\right)(z) .
$$

By 6.13, we have a homomorphism $\varphi: G(K) \rightarrow \Xi\left(\mathfrak{A}_{K}\right)$ extending, $\varphi_{0}, \varphi_{ \pm}$which is surjective since $j=t_{e} \circ \tilde{t}_{e} \circ t_{e}$. Assume that $\varphi(\exp (x) \exp (y) h \cdot \exp (z))=$ $t_{x} \circ \tilde{t}_{y} \circ g \circ t_{z}=\mathrm{Id}$ (where $h=\left(g, g^{\#-1}\right)$ ). Then $\tilde{t}_{y} \circ g=t_{-x-z}$, therefore $0=$ $\tilde{t}_{y}(0)=t_{-x-z} \circ g^{-1}(0)=-x-z$, hence $g^{-1}=\tilde{t}_{y}=j \circ t_{y} \circ j$, and $t_{y}=j \circ g^{-1} \circ j$ $=g^{\sharp}$ which implies $0=g^{\sharp}(0)=t_{y}(0)=y$. Thus $g=\mathrm{Id}, h=1$, and

$$
\exp (x) \exp (y) h \cdot \exp (z)=\exp (x+z)=1 \text {. }
$$

This shows that $\varphi$ is an isomorphism. By 6.12, $G(k)=U^{+}(k) U^{-}(k) H(k) U^{+}(k)$. It follows that $\varphi$ maps $G(k)$ isomorphically onto $\Xi(\mathfrak{U})$. Also we see that every element of $E(\mathfrak{U})$ is of the form $g \circ t_{x} \circ j \circ t_{y} \circ j \circ t_{z}$ with $g \in \operatorname{Str}(\mathfrak{U}), x$, $y, z \in \mathfrak{A}$. This was proved by Koecher in case $k$ is infinite and of charcteristic $\neq 2$.

Added in proof. In the paper O. Loos, Homogeneous algebraic varieties defined by Jordan pairs. Mh. Math. 86 (1978), 107-127, some of the results of $\S 6$ are extended to the case of an arbitrary base ring.

\section{BiBLIOGRAPHY}

[1] S. Anantharaman, Schémas en groupes, espaces homogènes, et espaces algébriques sur une base de dimension 1. Bull. Soc. Math. France, Meroire 33 (1973), 5-79.

[2] A. Borel, Linear algebraic groups. W.A. Benjamin, New York-Amsterdam 1969.

[ 3 ] A. Borel and J. Tits, Groupes réductifs. Publ. Math. IHES No. 27 (1965), 55-150.

[ 4 ] - Homomorphismes "abstraits" de groupes algébriques simples. Ann. of Math. 97 (1973), 499-571.

[ 5 ] M. Demazure and P. Gabriel, Groupes algébriques. Masson, Paris 1970. 
[6] J. Faulkner, Octonion planes defined by quadratic Jordan algebras. Memoirs Amer. Math. Soc. No. 104, 1970.

[ 7 ] A. Grothendieck and M. Demazure, Schémas en groupes. Springer Lecture notes 151-153, 1970. (Referred to as SGA3)

[ 8 ] A. Grothendieck and J. Dieudonné, Eléments de géometrie algébrique. Publ. Math. IHES 1960-.... (Referred to as EGA)

[9] S. Helgason, Differential Geometry and Symmetric Spaces. Academic Press, 1962.

[10] M. Koecher, Imbedding of Jordan algebras into Lie algebras I, II. Amer. J. Math. 89 (1967), 787-816, and 90 (1968), 476-510.

[11] _ U Über eine Gruppe von rationalen Abbildungen. Inv. Math. 3 (1967), 136171.

[12] — Gruppen und Lie-Algebren von rationalen Funktionen. Math. Z. 109 (1969), $349-392$.

[13] —, An elementary approach to bounded symmetric domains. Lecture notes, Rice University, Houston 1969.

[14] O. Loos, Representations of Jordan triples. Trans. Amer. Math. Soc. 185 (1973), 199-211.

[15] — Jordan pairs. Springer Lecture notes No. 460, 1975.

[16] - Jordan pairs and bounded symmetric domains. Lecture notes, University of California, Irvine 1977.

[17] K. Meyberg, Jordan-Tripelsysteme und die Koecher-Konstruktion von Lie-Algebren. Math. Z. 115 (1970), 58-78.

[18] H. P. Petersson, Zur Arithmetik der Jordan-Paare. Math. Z. 147 (1976), 139161.

[19] N. Roby, Lois polynomes et lois formelles en theorie des modules. Ann. Scient. Ecole Norm. Sup. $3^{\mathrm{e}}$ serie, t. 80 (1963), 213-348.

[20] T. A. Springer, Jordan algebras and algebraic groups. Springer Verlag 1973.

[21] J. Tits, Une classe d'algèbres de Lie en relation avec les algèbres de Jordan. Pruc. Kon. Akad. Wet. Amst. 65 (1962), 530-535.

Department of Mathematics

University of British Columbia

Current address :

Institut für Mathematik

Universität Innsbruck

Innsbruck, Austria 